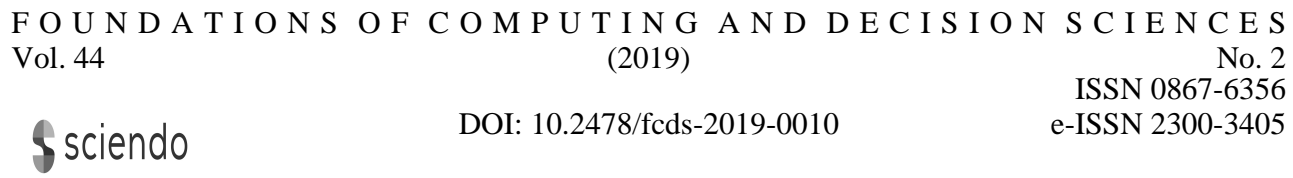

\title{
Toward an Efficient Resolution for a Single-machine Bi-objective Scheduling Problem with Rejection
}

\author{
Atefeh Moghaddam *, Jacques Teghem, Daniel Tuyttens `ं, \\ Farouk Yalaoui, Lionel Amodeo $\ddagger$
}

\begin{abstract}
We consider a single-machine bi-objective scheduling problem with rejection. In this problem, it is possible to reject some jobs. Four algorithms are provided to solve this scheduling problem. The two objectives are the total weighted completion time and the total rejection cost. The aim is to determine the set of efficient solutions. Four heuristics are described; they are implicit enumeration algorithms forming a branching tree, each one having two versions according to the root of the tree corresponding either to acceptance or rejection of all the jobs. The algorithms are first illustrated by a didactic example. Then they are compared on a large set of instances of various dimension and their respective performances are analysed.
\end{abstract}

Keywords: Production scheduling, bi-objective optimization, single-machine, rejection cost.

\section{Introduction}

The majority of the researches in the scheduling problem literature [2] considered that all the jobs must be scheduled in the workshop. However, there are particular practical situations where all the jobs may not be accepted and thus some of them rejected for specific reasons as congestion of the workshop, impossibility to meet customers requirements or as very long processing time or less importance of a job.

*Siemens Mobility SAS, 150 Avenue de la République, 92323 Chatillon Cedex France, e-mail: atefeh.moghaddam@gmail.com

${ }^{\dagger}$ Mathematics and Operational Research Unit, University of Mons, Polytechnic Faculty, Belgium, e-mail: \{jacques.teghem,daniel.tuyttens\}@umons.ac.be

$\ddagger$ Charles Delaunay Institute (ICD-LOSI), University of Technology of Troyes, France, e-mail: \{farouk.yalaoui, lionel.amodeo\}@utt.fr 
In such a case, the rejection of a job induces a penalty which can be considered like an outsourcing cost or an opportunity loss.

To our knowledge, the paper of Bartel et al. in 2000 [1] is the first to introduce the possible rejection of a job. After this initial study, several authors were interested to propose specific situations of scheduling problems with rejection of jobs. Their works are listed in the paper of Moghaddam et al. [8]. The majority of them analyse singleobjective problems where the rejection cost is aggregated with a classical scheduling criterion or the rejection cost is limited by a constraint. Researches on the rejection cost as a separate objective in a multi-objective problem is still rare in the literature [9]. That is the reason why we address the specific problem in this paper.

Moreover, except the papers of Moghaddam et al. [6], [7] and [8], even in a biobjective context, none of these works proposes algorithms to determine the set of efficient solutions. It is also the case in two most recent studies: the one of Wang et al. [11] where the authors introduce a special mechanism to control the processing times and the one of Zhang et al. [12] in which the off-line and the on-line two machines flow-shop scheduling problems with rejection are analysed.

An exception is the paper of Jia et al. [5]: some ant colony optimization (ACO) metaheuristics are proposed to find the Pareto efficient solutions set for the bi-criteria optimization of the makespan and the total rejection cost in a batch scheduling model on parallel machines.

Using the formalism of T'Kindt and Billaut [9], the model analysed in the present paper can be denoted by $1 / /\left(C_{w}, R\right)$, where $C_{w}$ is the total weighted completion time of the accepted jobs and $R$ is the total cost of the rejected jobs, and it consists to determine the set of efficient solutions for these two criteria. This model has been first introduced by Moghaddam et al. In [7], various simulated annealing metaheuristics are compared to obtain the efficient solutions based on the Pareto dominance. In [6], these algorithms are also analysed but based on the Lorenz dominance. Both dominance concepts are also considered in [8] where the results of two main metaheuristics, MOSA [10] and NSGAII [3] are compared.

The same model with Pareto dominance is analysed in the present paper, but dedicated implicit enumeration heuristics are developed instead of general metaheuristics. The rest of the paper is organized as follows. In Section 2, we define the problem in more details and we introduce in Section 3 some concepts used in the algorithms. The first four sub-sections of Section 4 describe respectively four implicit enumeration heuristics $H 1, H 2, H 3$ and $H 4$, each one having two versions according to the root of the branching tree corresponding either to the acceptance or the rejection of all the jobs. These eight heuristics are illustrated with a didactic example and in the sub-section 4.5, further comparison between them is illustrated. In Section 5, a large set of instances, with the number of jobs varying from 5 till 200, are solved with all the heuristics and a detailed analysis of their respective results is presented. The practical implementation of the algorithms is commented in sub-section 5.4. Section 6 provides a short conclusion of the study. 


\section{The problem}

We consider a single-machine scheduling problem. There are $n$ jobs and each job $j \in\{1, \ldots, n\}$ is characterized by its processing time $p_{j}$ and its own weight $w_{j}$.

A first objective is to minimize the total weighted completion time $C_{w}$, for which it is well known [2] that an optimal solution consists to schedule the jobs in the increasing order of the ratio $p_{j} / w_{j}$. For this reason, we assume that the $n$ jobs are initially ranked in this order:

$$
p_{1} / w_{1} \leq p_{2} / w_{2} \leq \ldots \leq p_{n} / w_{n}
$$

Nevertheless, it is possible to decide not to schedule all the jobs and thus to reject some of them. If the job $j \in\{1, \ldots, n\}$ is rejected, a rejection cost or penalty $r_{j}$ is incurred. A second objective $R$ is to minimize the total rejection cost i.e. the sum of the penalties $r_{j}$ of the rejected jobs. A solution $s$ is characterized by $A \subseteq\{1, \ldots, n\}$ the set of rejected jobs and by $\bar{A}=\{1, \ldots, n\} \backslash A$ the set of accepted jobs scheduled in the order (1).

The two considered objectives to minimize are thus

$$
\begin{aligned}
C_{w}(s) & =\sum_{j \in \bar{A}} w_{j} C_{j} \\
R(s) & =\sum_{j \in A} r_{j}
\end{aligned}
$$

where $C_{j}$ is the completion time of job $j \in \bar{A}$ in the optimal schedule of the jobs in subset $\bar{A}$ obtained by the order of relation (1).

Let us note that as

$$
C_{j}=\sum_{\substack{k \in \bar{A} \\ k \leq j}} p_{k}
$$

relation (2) can be written equivalently

$$
C_{w}(s)=\sum_{j \in \bar{A}} p_{j}\left(\sum_{\substack{k \in \bar{A} \\ k \geq j}} w_{k}\right)
$$

For this bi-objective scheduling problem, our aim is to determine (or to approximate) the set of efficient solutions and the corresponding Pareto front.

We recall [9] that a feasible solution $s$ is efficient if it does not exist any other feasible solution $s^{\prime}$ such that

$$
\begin{aligned}
C_{w}\left(s^{\prime}\right) & \leq C_{w}(s) \\
R\left(s^{\prime}\right) & \leq R(s)
\end{aligned}
$$

with at least one strict inequality.

We denote by $E$ the set of efficient solutions. The points $\left\{\left(C_{w}(s), R(s)\right) \mid s \in E\right\}$ correspond to the non-dominated points in the bi-objective space and form the so called Pareto front. 
Obviously the two extreme efficient solutions, denoted by $s_{a}$ and $s_{b}$, correspond respectively to $A=\emptyset$ and $A=\{1, \ldots, n\}$, giving the two extreme points of the Pareto front $\left(\bar{C}_{w}=C_{w}\left(s_{a}\right)=\sum_{j=1}^{n} w_{j} C_{j}, \underline{R}=R\left(s_{a}\right)=0\right)$ and $\left(\underline{C}_{w}=C_{w}\left(s_{b}\right)=0, \bar{R}=\right.$ $\left.R\left(s_{b}\right)=\sum_{j=1}^{n} r_{j}\right)$. For all efficient solutions $s \in E$, we have thus

$$
\begin{gathered}
\underline{C}_{w}=C_{w}\left(s_{b}\right)=0 \leq C_{w}(s) \leq C_{w}\left(s_{a}\right)=\bar{C}_{w} \\
\underline{R}=R\left(s_{a}\right)=0 \leq R(s) \leq R\left(s_{b}\right)=\bar{R}
\end{gathered}
$$

\section{Terminology}

\subsection{Potential efficient solution}

In the algorithms proposed in this paper, in a certain iteration, a solution is called a "potential efficient solution" if it is not dominated by any other solutions.

The set of potential efficient solutions is denoted by $P E$. Each time new solutions are generated, the list $P E$ is updated.

\subsection{Algorithms of type $a$ and type $b$}

A solution $s$ is of level $k$, if the number of rejected jobs is equal to $k$, i.e. if $|A|=k$. The algorithms in Section 4 are implicit enumeration forming a tree. An algorithm is said of type $a$, if the root of the tree is the solution $s_{a}$ (i.e. the unique solution of level 0 ) and the branching scheme increases progressively the considered level, creating children solutions at level $k+1$ by rejecting an additional job from a parent solution at level $k$.

An algorithm is said of type $b$, if the root of the tree is the solution $s_{b}$ (i.e. the unique solution of level $n$ ) and the branching scheme decreases progressively the considered level, creating children solutions at level $k-1$ by the acceptance of an additional job from a parent solution at level $k$.

For both types, the considered children solutions of a parent solution $s$ will be denoted by $E(s)$.

\subsection{Children nodes generated by non-dominated variations}

\subsubsection{Algorithm of type $a$}

At each level, the branching scheme creating $E(s)$ is based on a dominance relation defined by decreasing the first objective value $C_{w}$ and increasing the second objective value $R$. 


\section{$E\left(s_{a}\right)$ at level 1}

The parent of this solution is the initial solution $s_{a}$ at level 0 with $C_{w}\left(s_{a}\right)=\bar{C}_{w}$ and $R\left(s_{a}\right)=0$.

If a child solution $s_{1}$ at level 1 is characterized by $A=\{j\}$, using relation (5), it is easy to see that

$$
\begin{aligned}
C_{w}\left(s_{1}\right) & =\bar{C}_{w}-\Delta_{j}^{(1)} \\
R\left(s_{1}\right) & =0+r_{j}
\end{aligned}
$$

where

$$
\Delta_{j}^{(1)}=p_{j}\left(\sum_{k=j}^{n} w_{k}\right)+w_{j}\left(\sum_{l=1}^{j-1} p_{l}\right)
$$

Definition: Dominance relation of variation $\left(\Delta_{j}^{(1)}, r_{j}\right)$

In an algorithm of type $a$, the variation $\left(\Delta_{j}^{(1)}, r_{j}\right)$ is dominated if there exists another job $k \in\{1, \ldots, n\} \backslash\{j\}$ such that

$$
\begin{aligned}
\Delta_{k}^{(1)} & \geq \Delta_{j}^{(1)} \\
r_{k} & \leq r_{j}
\end{aligned}
$$

with at least one strict inequality.

In such a case, by rejecting job $k$, it is possible to have a larger decrease of the objective $C_{w}$ at a smaller increase of the objective $R$. It is clear that the nondominated variations are the only onces able to possibly generate an efficient solution at level 1.

So the branching scheme will only generate the set of children solutions $E\left(s_{a}\right)$ with each solution $s_{1}$ corresponding to a non-dominated variation $\left(\Delta_{j}^{(1)}, r_{j}\right)$.

At this first iteration, all the solutions $E\left(s_{a}\right)$ are potential efficient solutions and there are all assigned in the list $P E$.

\section{$E(s)$ at level $k=2, \ldots, n$}

From a parent solution $s_{k-1}$, with $A=\left\{j_{1}, \ldots, j_{k-1}\right\}$ at level $k-1$, an additional job $j$ can be rejected to build a child solution $s_{k}$ with $A=\left\{j_{1}, \ldots, j_{k-1}, j\right\}$.

Using relation (5), the variation of the two objectives is iteratively determined:

$$
\begin{aligned}
C_{w}\left(s_{k}\right) & =C_{w}\left(s_{k-1}\right)-\Delta_{j}^{(k)} \\
R\left(s_{k}\right) & =R\left(s_{k-1}\right)+r_{j}
\end{aligned}
$$

where

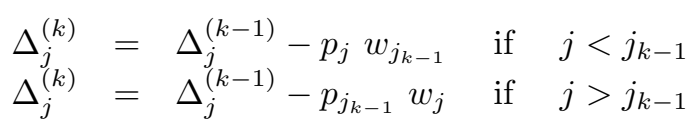


The non-dominated variations $\left(\Delta_{j}^{(k)}, r_{j}\right)$ are the only ones able to possibly generate potential efficient solutions $s_{k}$. So the branching scheme will only generate the set of children $E\left(s_{k-1}\right)$ with each solution $s_{k}$ corresponding to a non-dominated variation $\left(\Delta_{j}^{(k)}, r_{j}\right)$.

Remark 1: At each level $k$, the set of non-dominated variations $\left(\Delta_{j}^{(k)}, r_{j}\right)$ is always non-empty, containing at least the job $j$ with the smallest $r_{j}$ and the job $j$ with the largest $\Delta_{j}^{(k)}$. If it is the same job, then there exists only one non-dominated variation.

\subsubsection{Algorithm of type $b$}

When an algorithm of type $b$ is considered, with the root $s_{b}$ at level $n$, with $C_{w}\left(s_{b}\right)=0$ and $R\left(s_{b}\right)=\bar{R}$, at each level the branching scheme creating $E(s)$ consists to schedule an additional job and is also based on a dominance relation defined by the increase of the first objective $C_{w}$ and the decrease of the second objective $R$.

The concept is similar and the equations $(6),(7),(8),(9)$ and (10), are replaced by the following equations $(11),(12),(15),(13)$ and (14).

$$
\begin{aligned}
C_{w}\left(s_{n-1}\right) & =\Delta_{j}^{(n-1)} \\
R\left(s_{n-1}\right) & =\bar{R}-r_{j}
\end{aligned}
$$

where

$$
\begin{gathered}
\Delta_{j}^{(n-1)}=p_{j} w_{j} \\
C_{w}\left(s_{k}\right)=C_{w}\left(s_{k+1}\right)+\Delta_{j}^{(k)} \\
R\left(s_{k}\right)=R\left(s_{k+1}\right)-r_{j}
\end{gathered}
$$

where

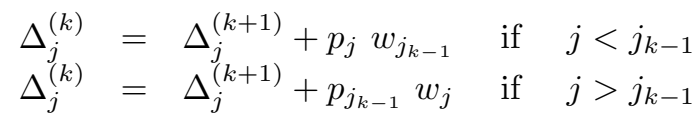

with $s_{k+1}$ characterized by $\bar{A}=\left\{j_{1}, \ldots, j_{k-1}\right\}$ and $s_{k}$ by $\bar{A}=\left\{j_{1}, \ldots, j_{k-1}, j\right\}$.

The dominance relation of variation $\left(\Delta_{j}^{(1)}, r_{j}\right)$ could be defined as following:

$$
\begin{aligned}
\Delta_{k}^{(n-1)} & \leq \Delta_{j}^{(n-1)} \\
r_{k} & \geq r_{j}
\end{aligned}
$$

with at least one strict inequality.

As for the algorithm of type $a$, the branching scheme will only generate the set of children $E\left(s_{k+1}\right)$ with each solution $s_{k}$ corresponding to a non-dominated variation $\left(\Delta_{j}^{(k)}, r_{j}\right)$. 


\section{Algorithms $H 1, H 2, H 3$ and $H 4$}

Each of these four algorithms can be of type $a$ or type $b$. In these algorithms, we denote by $P$ the pool of solutions which must be still considered by the branching scheme. These algorithms will be illustrated on the following didactic example with 5 jobs (already in the order of increasing ratio $p_{j} / w_{j}$ ).

Table 1: Data of the didactic example

\begin{tabular}{c|ccccc}
$j$ & 1 & 2 & 3 & 4 & 5 \\
\hline$p_{j}$ & 7 & 5 & 10 & 8 & 20 \\
$w_{j}$ & 8 & 4 & 6 & 4 & 2 \\
\hline$r_{j}$ & 5 & 1 & 5 & 3 & 2
\end{tabular}

\subsection{Algorithms $H 1$}

\subsection{1. $\quad H 1 a$}

In this algorithm, to describe a solution we will assign a status to each job with one of the three possibilities:

$$
\begin{array}{lcl}
\text { status } & 0 & \text { scheduled job free to be rejected, } \\
\text { status } & -1 & \text { definitively rejected job, } \\
\text { status } & 1 & \text { definitively scheduled job. }
\end{array}
$$

The initial solution is $s_{a}=(0, \ldots, 0)$ and assigned to the pool $P$. The solutions of $P$ are ranked in the increasing order of the objective $R$; in case of equality of this objective, the solutions are sorted in the order of their generation (see remark 2 below). When a new solution is examined by the branching scheme, the first solution of $P$ is chosen and this solution is removed. The algorithm comes to the end when $P=\emptyset$.

When a set of children solutions is created, they are compared with those of $P E$. Only the new potential solutions are assigned to the pool $P$, the others are fathomed in the tree, i.e. they are no more considered for the branching scheme.

The aim of the status "1" of a job is to avoid non-empty intersections between the sets of solutions corresponding to the different non-fathomed branches of the tree. For instance at the first iteration of $H 1 a$ on the didactic example where the children solutions consist to reject one of the three jobs 2,4 or 3 , these three children solutions will be $(0,-1,0,0,0),(0,1,0,-1,0)$ and $(0,1,-1,1,0)$.

The rejection of job 2, considered in the first branch, will no more be examined in the second and third branches, and the rejection of job 4, considered in the second branch, will no more be examined in the third branch. 
Table 2: Iterations of algorithm $H 1 a$ on the didactic example

\begin{tabular}{|c|c|c|c|c|c|c|}
\hline$I t$ & $s$ & $E(s)$ & $\left(R, C_{w}\right)$ & Level & $P$ & $P E$ \\
\hline 0 & & $s_{0}=(0,0,0,0,0)$ & $(0,456)$ & 0 & $s_{0}$ & $s_{0}$ \\
\hline 1 & $s_{0}$ & $\begin{array}{l}s_{1}=(0,-1,0,0,0) \\
s_{3}=(0,1,0,-1,0) \\
s_{5}=(0,1,-1,1,0)\end{array}$ & $\begin{array}{l}(1,348) \\
(3,320) \\
(5,264)\end{array}$ & $\begin{array}{l}1 \\
1 \\
1\end{array}$ & $\begin{array}{l}s_{1}, s_{3} \\
s_{5}\end{array}$ & $s_{0}, s_{1}, s_{3}, s_{5}$ \\
\hline 2 & $s_{1}$ & $\begin{array}{l}s_{3}^{2}=(0,-1,0,0,-1) \\
s_{4}=(0,-1,0,-1,1) \\
s_{6}=(0,-1,-1,1,1)\end{array}$ & $\begin{array}{l}(3,258) \\
(4,232) \\
(6,186)\end{array}$ & $\begin{array}{l}2 \\
2 \\
2\end{array}$ & $\begin{array}{l}s_{3}, s_{3}^{2} \\
s_{4}, s_{5} \\
s_{6} \\
\end{array}$ & $\begin{array}{l}s_{0}, s_{1}, s_{3}^{2}, s_{4} \\
s_{6}\end{array}$ \\
\hline 3 & $s_{3}$ & $\begin{array}{l}s_{5}^{2}=(0,1,0,-1,-1) \\
s_{8}=(0,1,-1,-1,0)\end{array}$ & $\begin{array}{l}(5,236) \\
(8,168)\end{array}$ & $\begin{array}{l}2 \prec s_{4} \\
2\end{array}$ & $\begin{array}{l}s_{3}^{2}, s_{4} \\
s_{5}, s_{6} \\
s_{8}\end{array}$ & $\begin{array}{l}s_{0}, s_{1}, s_{3}^{2}, s_{4} \\
s_{6}, s_{8}\end{array}$ \\
\hline 4 & $s_{3}^{2}$ & $\begin{array}{l}s_{6}^{2}=(0,-1,0,-1,-1) \\
s_{8}^{2}=(0,-1,-1,1,-1)\end{array}$ & $\begin{array}{l}(6,158) \\
(8,116)\end{array}$ & $\begin{array}{l}3 \\
3\end{array}$ & $\begin{array}{l}s_{4}, s_{5} \\
s_{6}, s_{6}^{2} \\
s_{8}, s_{8}^{2}\end{array}$ & $\begin{array}{l}s_{0}, s_{1}, s_{3}^{2}, s_{4} \\
s_{6}^{2}, s_{8}^{2}\end{array}$ \\
\hline 5 & $s_{4}$ & $s_{9}=(0,-1,-1,-1,1)$ & $(9,110)$ & 3 & $\begin{array}{l}s_{5}, s_{6} \\
s_{6}^{2}, s_{8} \\
s_{8}^{2}, s_{9}\end{array}$ & $\begin{array}{l}s_{0}, s_{1}, s_{3}^{2}, s_{4} \\
s_{6}^{2}, s_{8}^{2}, s_{9}\end{array}$ \\
\hline 6 & $s_{5}$ & $\begin{array}{l}s_{7}=(0,1,-1,1,-1) \\
s_{10}=(-1,1,-1,1,0)\end{array}$ & $\begin{array}{c}(7,184) \\
(10,138)\end{array}$ & $\begin{array}{l}2 \prec s_{6}^{2} \\
2 \prec s_{9}\end{array}$ & $\begin{array}{l}s_{6}, s_{6}^{2} \\
s_{8}, s_{8}^{2} \\
s_{9}\end{array}$ & $\begin{array}{l}s_{0}, s_{1}, s_{3}^{2}, s_{4} \\
s_{6}^{2}, s_{8}^{2}, s_{9}\end{array}$ \\
\hline 7 & $s_{6}$ & $s_{11}=(-1,-1,-1,1,1)$ & $(11,88)$ & 3 & $\begin{array}{l}s_{6}^{2}, s_{8} \\
s_{8}^{2}, s_{9}\end{array}$ & $\begin{array}{l}s_{0}, s_{1}, s_{3}^{2}, s_{4} \\
s_{6}^{2}, s_{8}^{2}, s_{9}, s_{11}\end{array}$ \\
\hline 8 & $s_{6}^{2}$ & $s_{11}^{2}=(0,-1,-1,-1,-1)$ & $(11,56)$ & 4 & $\begin{array}{l}s_{8}, s_{8}^{2} \\
s_{9}, s_{11}^{2}\end{array}$ & $\begin{array}{l}s_{0}, s_{1}, s_{3}^{2}, s_{4} \\
s_{6}^{2}, s_{8}^{2}, s_{9}, s_{11}^{2}\end{array}$ \\
\hline 9 & $s_{8}$ & $\begin{array}{l}s_{10}^{2}=(0,1,-1,-1,-1) \\
s_{13}=(-1,1,-1,-1,0)\end{array}$ & $\begin{array}{c}(10,104) \\
(13,70)\end{array}$ & $\begin{array}{l}3 \\
3 \prec s_{11}^{2}\end{array}$ & $\begin{array}{l}s_{8}^{2}, s_{9} \\
s_{10}^{2}, s_{11}^{2}\end{array}$ & $\begin{array}{l}s_{0}, s_{1}, s_{3}^{2}, s_{4} \\
s_{6}^{2}, s_{8}^{2}, s_{9}, s_{10}^{2} \\
s_{11}^{2}\end{array}$ \\
\hline 10 & $s_{8}^{2}$ & $s_{13}^{2}=(-1,-1,-1,1,-1)$ & $(13,32)$ & 4 & $\begin{array}{l}s_{9}, s_{10}^{2} \\
s_{11}^{2}\end{array}$ & $\begin{array}{l}s_{0}, s_{1}, s_{3}^{2}, s_{4} \\
s_{6}^{2}, s_{8}^{2}, s_{9}, s_{10}^{2} \\
s_{11}^{2}, s_{13}^{2}\end{array}$ \\
\hline 11 & $s_{9}$ & $s_{14}=(-1,-1,-1,-1,1)$ & $(14,40)$ & $4 \prec s_{13}^{2}$ & $s_{10}^{2}, s_{11}^{2}$ & $\begin{array}{l}s_{0}, s_{1}, s_{3}^{2}, s_{4} \\
s_{6}^{2}, s_{8}^{2}, s_{9}, s_{10}^{2} \\
s_{11}^{2}, s_{13}^{2}\end{array}$ \\
\hline 12 & $s_{10}^{2}$ & $s_{15}=(-1,1,-1,-1,-1)$ & $(15,20)$ & 4 & $s_{11}^{2}$ & $\begin{array}{l}s_{0}, s_{1}, s_{3}^{2}, s_{4} \\
s_{6}^{2}, s_{8}^{2}, s_{9}, s_{10}^{2} \\
s_{11}^{2}, s_{13}^{2}, s_{15} \\
\end{array}$ \\
\hline 13 & $s_{11}^{2}$ & $s_{16}=(-1,-1,-1,-1,-1)$ & $(16,0)$ & 5 & & $\begin{array}{l}s_{0}, s_{1}, s_{3}^{2}, s_{4} \\
s_{6}^{2}, s_{8}^{2}, s_{9}, s_{10}^{2} \\
s_{11}^{2}, s_{13}^{2}, s_{15}, s_{16} \\
\end{array}$ \\
\hline
\end{tabular}




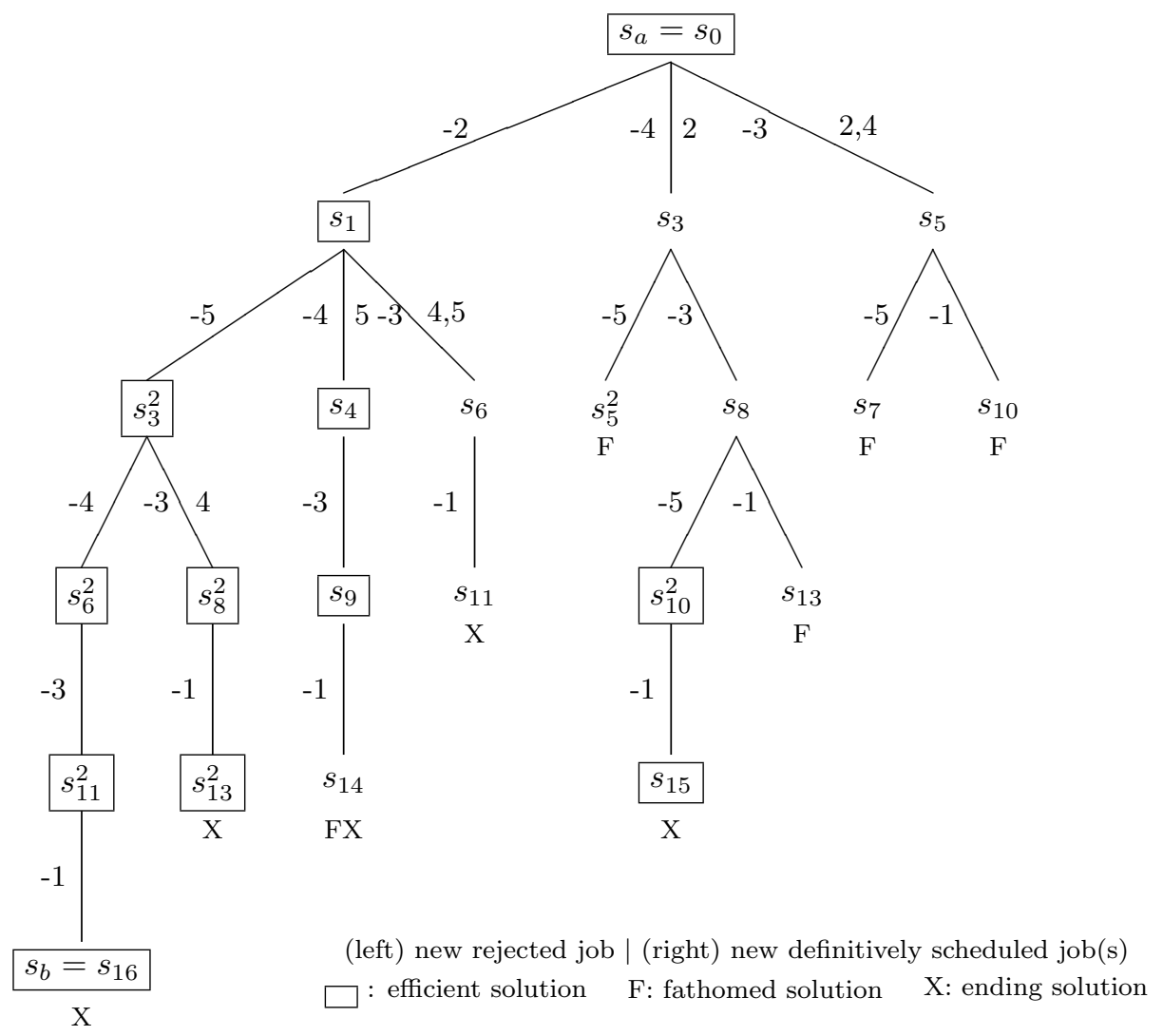

Figure 1: Branching scheme of $H 1 a$

For a solution with no job with the status "0", this solution is an ending solution of the branch. Table 2 describes the successive iterations of the algorithm $H 1 a$ on the didactic example. Figure 1 represents the corresponding branching scheme. The twelve efficient solutions of this instance are generated. For this didactic example, the subindex of a solution is the value for the objective $R$; in case of identical value, the upper index indicates the order of their generation.

\section{Remark 2: An impact of the order of the solutions in the pool $P$}

Let us suppose that inside the pool $P$, the solutions with identical value of objective $R$ are ordered in the increasing order of objective $C_{w}$ in contrary to the order of their generation. Table 3 indicates what happens for the didactic example with this change. The solution $s_{3}^{2}$, with $C_{w}\left(s_{3}^{2}\right)=258$, will be treated before the solution $s_{3}$, with $C_{w}\left(s_{3}\right)=320$. So the solution $s_{8}^{2}$, a child solution of $s_{3}^{2}$, will be generated before the solution $s_{8}$, a child solution of $s_{3}$, and as $s_{8}$ is dominated by $s_{8}^{2}$, the solution $s_{8}$ will now be fathomed and no more included in the pool $P$. The consequence is (see iteration 9 of Table 2 and also Figure 1) that it will be impossible to still generate the efficient solution $s_{10}^{2}$ and so the efficient solution $s_{15}$, a child of $s_{10}^{2}$ (see iteration 
12 of Table 2).

So it appears that with such a change in the order to treat the solutions of the pool, only 10 efficient solutions will be generated instead of 12 . We have seen that this case can happen in several instances and it is the reason why we have chosen to order the solutions of the pool as indicated before.

Table 3: Impact of the lexicographic order

\begin{tabular}{|c|c|l|l|l|l|l|}
\hline$I t$ & $s$ & $E(s)$ & $\left(R, C_{w}\right)$ & Level & $P$ & $P E$ \\
\hline \hline \multirow{2}{*}{2} & \multirow{2}{*}{$s_{1}$} & $s_{3}^{2}=(0,-1,0,0,-1)$ & $(3,258)$ & 2 & $s_{3}^{2}, s_{3}, s_{4}, s_{5}$ & $s_{0}, s_{1}, s_{3}^{2}, s_{4}$ \\
& & $s_{4}=(0,-1,0,-1,1)$ & $(4,232)$ & 2 & $s_{6}$ & $s_{6}$ \\
& & $s_{6}=(0,-1,-1,1,1)$ & $(6,186)$ & 2 & & \\
\hline \multirow{2}{*}{3} & $s_{3}^{2}$ & $s_{6}^{2}=(0,-1,0,-1,-1)$ & $(6,158)$ & 3 & $s_{3}, s_{4}, s_{5}, s_{6}^{2}$ & $s_{0}, s_{1}, s_{3}^{2}, s_{4}$ \\
& & $s_{8}^{2}=(0,-1,-1,1,-1)$ & $(8,116)$ & 3 & $s_{6}, s_{8}^{2}$ & $s_{6}^{2}, s_{8}^{2}$ \\
\hline \multirow{2}{*}{4} & \multirow{2}{*}{$s_{3}$} & $s_{5}^{2}=(0,1,0,-1,-1)$ & $(5,236)$ & $2 \prec s_{4}$ & $s_{4}, s_{5}, s_{6}^{2}, s_{6}$ & $s_{0}, s_{1}, s_{3}^{2}, s_{4}$ \\
& & $s_{8}=(0,1,-1,-1,0)$ & $(8,168)$ & $2 \prec s_{8}^{2}$ & $s_{8}^{2}$ & $s_{6}^{2}, s_{8}^{2}$ \\
\hline
\end{tabular}

\subsection{2. $H 1 b$}

Algorithm $H 1 b$ is similar to algorithm $H 1 a$. The unique difference is that this algorithm is of type $b$ so that the initial solution is thus the extreme efficient solution $s_{b}$, initializing the pool $P$.

There are two logical modifications in comparison to $H 1 a$. First, the solutions of $P$ are ranked in the decreasing order of the objective $R$; in case of equality of this objective, the solutions are sorted according to their generation. Nevertheless, the management of the pool $P$ is identical to the one of $H 1 a$ and thus the dominated children solutions are not placed in the pool $P$.

Secondly, in the description of a solution, the three following status of a job are used:

$$
\begin{array}{lcl}
\text { status } & 0 & \text { rejected job free to be scheduled, } \\
\text { status } & -1 & \text { definitively rejected job, } \\
\text { status } & 1 & \text { definitively scheduled job, }
\end{array}
$$

so that $s_{b}=(0, \ldots, 0)$.

But the aim of the status " 1 " is also to avoid non-empty intersections between the sets of solutions corresponding to the non-fathomed different branches of the tree.

Table 4 describes the successive iterations of the algorithm $H 1 b$ on the didactic example. Figure 2 represents the corresponding branching scheme. The twelve efficient solutions of this instance are again generated even if the way to obtain those solutions in Figure 1 is quite different to the one in Figure 2. 
Table 4: Iterations of algorithm $H 1 b$ on the didactic example

\begin{tabular}{|c|c|c|c|c|c|c|}
\hline It & $s$ & $E(s)$ & $\left(R, C_{w}\right)$ & Level & $P$ & $P E$ \\
\hline 0 & & $s_{16}=(0,0,0,0,0)$ & $(16,0)$ & 5 & $s_{16}$ & $s_{16}$ \\
\hline 1 & $s_{16}$ & $\begin{array}{l}s_{15}=(0,1,0,0,0) \\
s_{13}=(0,-1,0,1,0) \\
s_{11}=(1,-1,0,-1,0)\end{array}$ & $\begin{array}{l}(15,20) \\
(13,32) \\
(11,56)\end{array}$ & $\begin{array}{l}4 \\
4 \\
4\end{array}$ & $s_{15}, s_{13}, s_{11}$ & $s_{11}, s_{13}, s_{15}, s_{16}$ \\
\hline 2 & $s_{15}$ & $\begin{array}{l}s_{13}^{2}=(0,1,0,0,1) \\
s_{12}=(0,1,0,1,0) \\
s_{10}=(1,1,0,0,0)\end{array}$ & $\begin{array}{c}(13,70) \\
(12,72) \\
(10,104)\end{array}$ & $\begin{array}{l}3 \prec s_{13} \\
3 \prec s_{11} \\
3\end{array}$ & $s_{13}, s_{11}, s_{10}$ & $\begin{array}{l}s_{10}, s_{11}, s_{13}, s_{15} \\
s_{16}\end{array}$ \\
\hline 3 & $s_{13}$ & $\begin{array}{l}s_{11}^{2}=(0,-1,0,1,1) \\
s_{8}=(1,-1,0,1,0)\end{array}$ & $\begin{array}{l}(11,88) \\
(8,116)\end{array}$ & $\begin{array}{l}3 \prec s_{11} \\
3\end{array}$ & $s_{11}, s_{10}, s_{8}$ & $\begin{array}{l}s_{8}, s_{10}, s_{11}, s_{13} \\
s_{15}, s_{16}\end{array}$ \\
\hline 4 & $s_{11}$ & $\begin{array}{l}s_{9}=(1,-1,0,-1,1) \\
s_{6}=(1,-1,1,-1,-1)\end{array}$ & $\begin{array}{l}(9,110) \\
(6,158)\end{array}$ & $\begin{array}{l}3 \\
3 \\
\end{array}$ & $s_{10}, s_{9}, s_{8}$ & $\begin{array}{l}s_{6}, s_{8}, s_{9}, s_{10} \\
s_{11}, s_{13}, s_{15}, s_{16}\end{array}$ \\
\hline 5 & $s_{10}$ & $\begin{array}{l}s_{8}^{2}=(1,1,0,0,1) \\
s_{7}=(1,1,0,1,0) \\
s_{5}=(1,1,1,0,0)\end{array}$ & $\begin{array}{l}(8,168) \\
(7,184) \\
(5,236)\end{array}$ & $\begin{array}{l}2 \prec s_{8} \\
2 \prec s_{6} \\
2\end{array}$ & $s_{9}, s_{8}, s_{5}$ & $\begin{array}{l}s_{5}, s_{6}, s_{8}, s_{9} \\
s_{10}, s_{11}, s_{13}, s_{15} \\
s_{16}\end{array}$ \\
\hline 6 & $s_{9}$ & $s_{4}=(1,-1,1,-1,1)$ & $(4,232)$ & 2 & $s_{8}, s_{5}$ & $\begin{array}{l}s_{4}, s_{6}, s_{8}, s_{9} \\
s_{10}, s_{11}, s_{13}, s_{15} \\
s_{16}\end{array}$ \\
\hline 7 & $s_{8}$ & $\begin{array}{l}s_{6}^{2}=(1,-1,0,1,1) \\
s_{3}=(1,-1,1,1,0)\end{array}$ & $\begin{array}{l}(6,186) \\
(3,258)\end{array}$ & $\begin{array}{l}2 \prec s_{6} \\
2\end{array}$ & $s_{5}, s_{3}$ & $\begin{array}{l}s_{3}, s_{4}, s_{6}, s_{8} \\
s_{9}, s_{10}, s_{11}, s_{13} \\
s_{15}, s_{16}\end{array}$ \\
\hline 8 & $s_{5}$ & $\begin{array}{l}s_{3}^{2}=(1,1,1,0,1) \\
s_{2}=(1,1,1,1,0)\end{array}$ & $\begin{array}{l}(3,320) \\
(2,356)\end{array}$ & $\begin{array}{l}1 \prec s_{3} \\
1\end{array}$ & $s_{3}, s_{2}$ & $\begin{array}{l}s_{2}, s_{3}, s_{4}, s_{6} \\
s_{8}, s_{9}, s_{10}, s_{11} \\
s_{13}, s_{15}, s_{16}\end{array}$ \\
\hline 9 & $s_{3}$ & $s_{1}=(1,-1,1,1,1)$ & $(1,348)$ & 1 & $s_{2}$ & $\begin{array}{l}s_{1}, s_{3}, s_{4}, s_{6} \\
s_{8}, s_{9}, s_{10}, s_{11} \\
s_{13}, s_{15}, s_{16} \\
\end{array}$ \\
\hline 10 & $s_{2}$ & $s_{0}=(1,1,1,1,1)$ & $(0,456)$ & 0 & & $\begin{array}{l}s_{0}, s_{1}, s_{3}, s_{4} \\
s_{6}, s_{8}, s_{9}, s_{10} \\
s_{11}, s_{13}, s_{15}, s_{16} \\
\end{array}$ \\
\hline
\end{tabular}




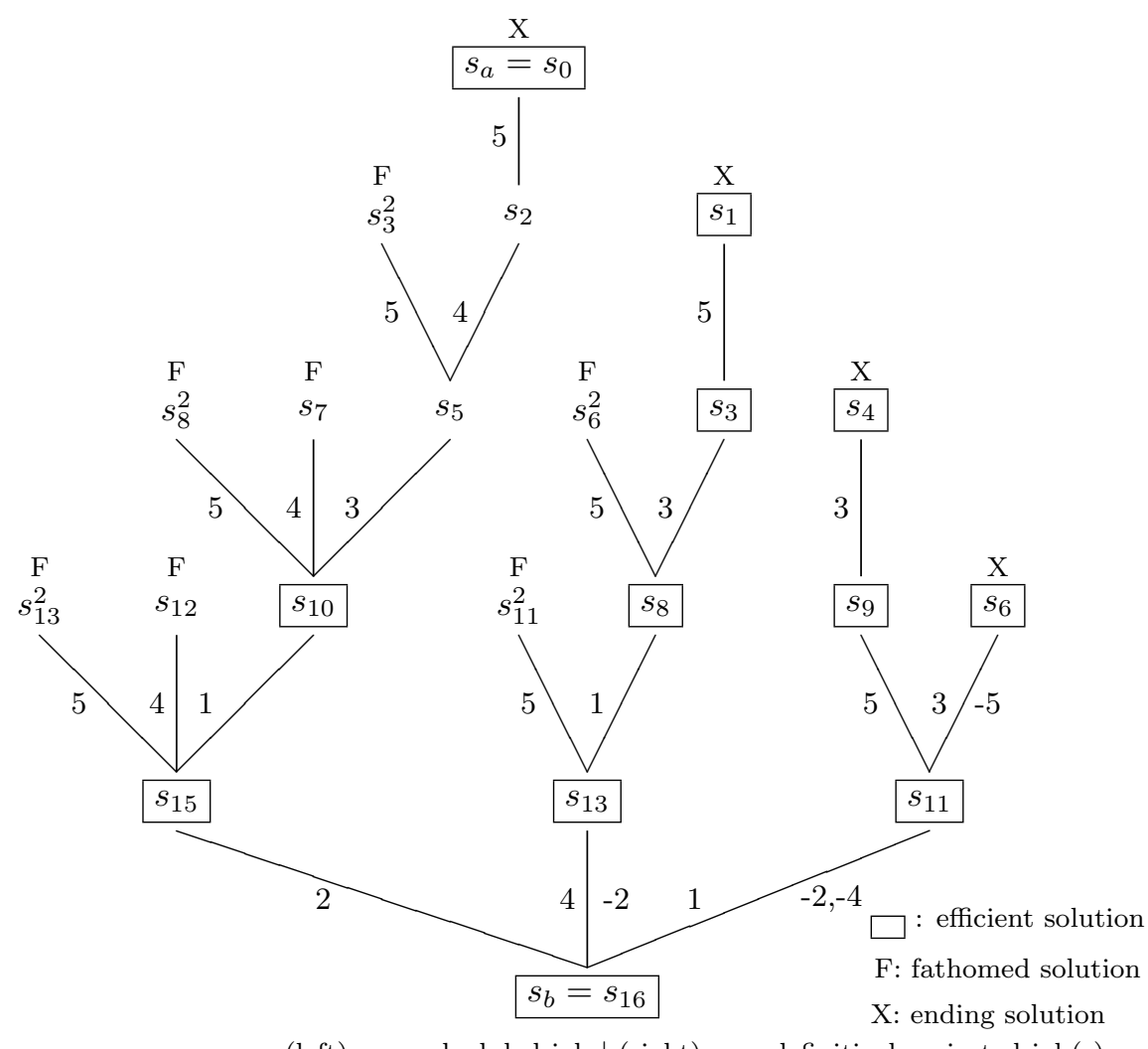

(left) new scheduled job | (right) new definitively rejected job(s)

Figure 2: Branching scheme of $H 1 b$

\subsection{3. $H 1 a b$}

As we will see in the numerical experiments of Section 5 , the two sets $P E$ obtained by $H 1 a$ and $H 1 b$, can be different, contrary to what happens in the didactic example. If these two sets are filtered by pairwise comparisons of the solutions, this filtering will be denoted by $H 1 a b$. Thus only the non-dominated solutions present in the union of these two sets are saved.

\subsection{Algorithms $H 2$}

\subsection{1. $\quad H 2 a$}

The algorithm $H 2 a$ presents a minor modification comparing to the algorithm $H 1 a$. As we will see in the numerical experiments (and see also above-mentioned remark 1), an efficient solution can be missed in the case where all the children solutions of the same parent solution are dominated and thus fathomed and not placed in the pool $P$ 
of solutions still to be examined.

It is the reason why in such a situation, in $H 2 a$ one of the dominated children solutions is kept in the pool $P$. To choose which solution to retain in the pool, different criteria are possible. For $H 2 a$, we decide to keep the dominated child solution with the minimum value of $R$ in the pool. Another possibility investigated is to keep the solution corresponding to the minimum ratio $r_{j} / \Delta_{j}$. In this case, the other dominated children solutions are fathomed.

In Table 5, an example of modification due to $H 2$ a comparing to Table 2 for $H 1 a$ is given. As the two children solutions $s_{7}$ and $s_{10}$ are dominated, the solution $s_{7}$ is kept in the pool.

Table 5: Algorithm $H 2 a$ when all children solutions are dominated solutions

\begin{tabular}{|c|c|l|c|l|l|l|}
\hline$I t$ & $s$ & $E(s)$ & $\left(R, C_{w}\right)$ & Level & $P$ & $P E$ \\
\hline \hline 6 & $s_{5}$ & $s_{7}=(0,1,-1,1,-1)$ & $(7,184)$ & $2 \prec s_{6}^{2}$ & $s_{6}, s_{6}^{2}, s_{7}, s_{8}$ & $s_{0}, s_{1}, s_{3}^{2}, s_{4}$ \\
& & $s_{10}=(-1,1,-1,1,0)$ & $(10,138)$ & $2 \prec s_{9}$ & $s_{8}^{2}, s_{9}$ & $s_{6}^{2}, s_{8}^{2}, s_{9}$ \\
\hline
\end{tabular}

\subsection{2. $\quad H 2 b$}

In $H 1 b$ a similar modification that the one made in $H 1 a$ is introduced. When all the children solutions of the same parent solution are dominated, one of them is kept in the pool $P$ : the dominated child solution with the maximal value of $R$ is assigned to the pool. The other dominated children solutions are fathomed.

\subsection{3. $H 2 a b$}

In this algorithm, the two sets of $P E$ obtained with $H 2 a$ and $H 2 b$ are filtered, keeping only the non-dominated solutions in the union of these two sets.

\subsection{Algorithms $H 3$}

\subsection{1. $\quad H 3 a$}

The principle of this algorithm is identical to the one $H 1 a$. The unique difference is that in $H 3 a$ the status " 1 " of a job is not considered. Thus there are only two possibilities for the status of a job:

$$
\begin{array}{lcl}
\text { status } & 0 & \text { scheduled job free to be rejected, } \\
\text { status } & -1 & \text { definitively rejected job. }
\end{array}
$$

In $H 1 a$, a job $j$ with the status " 1 " is no more considered to be rejected in the branching scheme so that its variation $\left(\Delta_{j}, r_{j}\right)$ is not taken into consideration. As a 
consequence, the set of non-dominated variations can be influenced by this fact. In $H 3 a$, all the variations for the jobs with " 0 " status are analyzed. This mechanism helps generating new branches, which can lead to efficient solutions ignored by $H 1 a$.

Of course, another consequence is that the intersection of the sets of solutions in non-fathomed branches are no more empty. The algorithm simply ignores redundant solutions, which have been generated in another branch.

The numerical experiments of Section 5 will prove that such a situation appears in the case of large-size instances.

Table 6 illustrates the first three iterations of $H 3 a$. We see that the non-dominated variations of iteration 3 are now obtained for jobs 2 and 3 , providing the solutions $s_{4}$ (already obtained) and $s_{8}$ instead in $H 1 a$ (see Table 2 ) for jobs 5 and 3 providing the solutions $s_{5}^{2}$ and $s_{8}$.

Table 6: Three first iterations of algorithm $H 3 a$ on the didactic example

\begin{tabular}{|c|c|c|c|c|c|c|}
\hline$I t$ & $s$ & $E(s)$ & $\left(R, C_{w}\right)$ & Level & $P$ & $P E$ \\
\hline 0 & & $\bar{s}_{0}=(0,0,0,0,0)$ & $\overline{(0,456)}$ & 0 & $s_{0}$ & $s_{0}$ \\
\hline 1 & $s_{0}$ & $\begin{array}{l}s_{1}=(0,-1,0,0,0) \\
s_{3}=(0,0,0,-1,0) \\
s_{5}=(0,0,-1,0,0)\end{array}$ & $\begin{array}{l}(1,348) \\
(3,320) \\
(5,264)\end{array}$ & $\begin{array}{l}1 \\
1 \\
1\end{array}$ & $s_{1}, s_{3}, s_{5}$ & $s_{0}, s_{1}, s_{3}, s_{5}$ \\
\hline 2 & $s_{1}$ & $\begin{array}{l}s_{3}^{2}=(0,-1,0,0,-1) \\
s_{4}=(0,-1,0,-1,0) \\
s_{6}=(0,-1,-1,0,0)\end{array}$ & $\begin{array}{l}(3,258) \\
(4,232) \\
(6,186) \\
\end{array}$ & $\begin{array}{l}2 \\
2 \\
2\end{array}$ & $\begin{array}{l}s_{3}, s_{3}^{2}, s_{4}, s_{5} \\
s_{6}\end{array}$ & $\begin{array}{l}s_{0}, s_{1}, s_{3}^{2}, s_{4} \\
s_{6}\end{array}$ \\
\hline 3 & $s_{3}$ & $\begin{array}{l}s_{4}=(0,-1,0,-1,0) \\
s_{8}=(0,0,-1,-1,0)\end{array}$ & $\begin{array}{l}(4,232) \\
(8,168)\end{array}$ & $\begin{array}{l}2 \longdiv { = s _ { 4 } } \\
2\end{array}$ & $\begin{array}{l}s_{3}^{2}, s_{4}, s_{5}, s_{6} \\
s_{8}\end{array}$ & $\begin{array}{l}s_{0}, s_{1}, s_{3}^{2}, s_{4} \\
s_{6}, s_{8}\end{array}$ \\
\hline
\end{tabular}

\subsection{2. $\quad H 3 b$}

A similar modification is made in $H 1 b$, i.e. the status "- 1 " of a job is no more considered, keeping only the two status:

status 0 rejected job free to be scheduled,

status 1 definitively scheduled job.

The consequences of this modification are identical to those described for $H 3 a$.

\subsection{3. $\quad H 3 a b$}

The result of this algorithm is the filtering of the two sets $H 3 a$ and $H 3 b$. 


\subsection{Algorithms $H 4$}

\subsection{1. $\quad H 4 a$}

In this algorithm, as in $H 3 a$, we do not consider the status "1" of a job, keeping only the status " 0 " and " -1 ". But here the status " 1 " means "rejected job free to be scheduled" but no more "definitively rejected job". This change differs the $H 4 a$ algorithm to $H 3, H 1$ and $H 2$ by one main element: if the solution parent is of level $k$, the possible children solutions will be of level $k-1$ or of level $k+1$. Effectively, the considered variations consist to change successively the status of each job; thus:

- the status "0" becomes "-1" generating solutions of level $k+1$,

- the status "-1" becomes "0" generating solutions of level $k-1$.

Among these $n$ variations, as in the three preceding algorithms, only the non-dominated variations are kept to define $E(s)$. Redundant solutions are ignored as they were in $H 3$ algorithm. Nevertheless, Table 7 illustrates this new branching scheme on the iterations 4 and 5 of Table 2 for the didactic example.

Table 7: First five iterations of algorithm $H 4 a$ on the didactic example

\begin{tabular}{|c|c|c|c|c|c|c|}
\hline$I t$ & $s$ & $E(s)$ & $\left(R, C_{w}\right)$ & Level & $P$ & $P E$ \\
\hline$\overline{00}$ & & 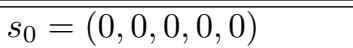 & $(0,456)$ & 0 & $s_{0}$ & $s_{0}$ \\
\hline 1 & $s_{0}$ & $\begin{array}{l}s_{1}=(0,-1,0,0,0) \\
s_{3}=(0,0,0,-1,0) \\
s_{5}=(0,0,-1,0,0)\end{array}$ & $\begin{array}{l}(1,348) \\
(3,320) \\
(5,264)\end{array}$ & $\begin{array}{l}1 \\
1 \\
1\end{array}$ & $s_{1}, s_{3}, s_{5}$ & $s_{0}, s_{1}, s_{3}, s_{5}$ \\
\hline 2 & $s_{1}$ & $\begin{array}{l}s_{3}^{2}=(0,-1,0,0,-1) \\
s_{4}=(0,-1,0,-1,0) \\
s_{6}=(0,-1,-1,0,0)\end{array}$ & $\begin{array}{l}(3,258) \\
(4,232) \\
(6,186)\end{array}$ & $\begin{array}{l}2 \\
2 \\
2\end{array}$ & $\begin{array}{l}s_{3}, s_{3}^{2}, s_{4}, s_{5} \\
s_{6}\end{array}$ & $\begin{array}{l}s_{0}, s_{1}, s_{3}^{2}, s_{4} \\
s_{6}\end{array}$ \\
\hline 3 & $s_{3}$ & $\begin{array}{l}s_{4}=(0,-1,0,-1,0) \\
s_{8}=(0,0,-1,-1,0)\end{array}$ & $\begin{array}{l}(4,232) \\
(8,168)\end{array}$ & $\begin{array}{l}2=s_{4} \\
2\end{array}$ & $\begin{array}{l}s_{3}^{2}, s_{4}, s_{5}, s_{6} \\
s_{8}\end{array}$ & $\begin{array}{l}s_{0}, s_{1}, s_{3}^{2}, s_{4} \\
s_{6}, s_{8}\end{array}$ \\
\hline 4 & $s_{3}^{2}$ & $\begin{array}{l}s_{2}=(0,0,0,0,-1) \\
s_{6}^{2}=(0,-1,0,-1,-1) \\
s_{8}^{2}=(0,-1,-1,0,-1)\end{array}$ & $\begin{array}{l}(2,356) \\
(6,158) \\
(8,116)\end{array}$ & $\begin{array}{l}1 \prec s_{1} \\
3 \\
3\end{array}$ & $\begin{array}{l}s_{4}, s_{5}, s_{6}, s_{6}^{2} \\
s_{8}, s_{8}^{2}\end{array}$ & $\begin{array}{l}s_{0}, s_{1}, s_{3}^{2}, s_{4} \\
s_{6}^{2}, s_{8}^{2}\end{array}$ \\
\hline 5 & $s_{4}$ & $\begin{array}{l}s_{3}=(0,0,0,0,-1) \\
s_{6}^{2}=(0,-1,0,-1,-1) \\
s_{9}=(0,-1,-1,-1,0)\end{array}$ & $\begin{array}{l}(3,320) \\
(6,158) \\
(9,110)\end{array}$ & $\begin{array}{l}1=s_{3} \\
3=s_{6}^{2} \\
3\end{array}$ & $\begin{array}{l}s_{5}, s_{6}, s_{6}^{2}, s_{8} \\
s_{8}^{2}, s_{9}\end{array}$ & $\begin{array}{l}s_{0}, s_{1}, s_{3}^{2}, s_{4} \\
s_{6}^{2}, s_{8}^{2}, s_{9}\end{array}$ \\
\hline
\end{tabular}

In the next section, the positive impact of $H 4$ on two larger instances is presented. 


\subsection{2. $H 4 b$}

By changing the initial efficient solution, algorithm $H 4 b$ can also be developed. Nevertheless, for all the instances treated in Section 5 , the results of $H 4 b$ will be systematically the same as those of $H 4 a$. For this reason, in the following, we will only mention $H 4$.

\subsection{Illustrations}

In this section, we will analyse the impact of the algorithms using the first two instances of Section 5 presenting some differences between the results of the algorithms. The data of these two instances 12 (13-3) and 13 (15-1), are given in Table 8 and Table 9 in the Annex.

\section{Instance $12(13-3)$}

This instance with 13 jobs has 41 efficient solutions. They are all generated by the algorithms $H 1 b, H 2 a, H 2 b, H 3 b$ and $H 4$. $H 1 a$ provides only 39 efficient solutions (see Table 10 in the Annex); the two missing solutions are:

- $s_{1}$ at level 8 , with rejected jobs $1,4,5,6,7,8,11$ and 12 ,

- $s_{2}$ at level 11, with rejected jobs $1,2,3,4,5,7,8,10,11,12$ and 13 .

$H 3 a$ provides 40 efficient solutions (see Table 14 in the Annex); $s_{2}$ is the missing solution.

\section{Remarks on the observations made.}

\section{$H 1 a$ versus $H 2 a$}

In $H 1 a$, at parent solution at level 6 corresponding to the successive rejects of jobs $7,12,5,4,8,6$ and the job 2 with status " 1 ", all the children solutions are dominated so that it is impossible to obtain $s_{1}$ corresponding to the reject of the additional jobs 1 and 11 .

But with $H 2 a$, the dominated child solution with the minimal rejection cost is kept in the pool; and during the two next iterations these two jobs are successively rejected providing $s_{1}$.

Similarly in $H 1 a$, at parent solution at level 9 corresponding to the successive rejects of jobs $7,12,5,4,8,2,11,1,10$ and the job 6 with status "1", all the children solutions are dominated and it is impossible to obtain $s_{2}$ corresponding to the reject of the additional jobs 3 and 13 . 
Again, with $H 2 a$, the dominated child solution with the minimal rejection cost is kept in the pool. This allows to reject jobs 3 and 13 during the two next iterations providing $s_{2}$.

\section{$H 3 a$ versus $H 4$}

In $H 3 a$, from the same parent solution at level 9 that was in $H 1 a$, it is impossible to obtain $s_{2}$.

It is useful to note that $s_{2}$ at level 11 is generated with $H 4$ from the parent solution at level 12 corresponding to the successive rejects of jobs $7,12,5,4,8,2,11,6,1,13,10,3$, changing the status "- 1 " of job 6 to status "0".

\section{Instance $13(15-1)$}

This instance with 15 jobs has 66 efficient solutions. They are all provided by algorithms $H 1 b, H 2 b, H 3 b$ and $H 4$. But $H 1 a, H 2 a$ and $H 3 a$ generate only 65 efficient solutions (see Table 10, Table 12 and Table 14).

\section{$H 3 a$ versus $H 4$}

It is important to note that the missing efficient solution at level 11 , corresponding to the reject of jobs $1,3,4,5,7,8,9,10,11,13$ and 14 is obtained by $H 4$ as child solution of a solution at level 12, changing the status "-1" of job 12 to status "0", despite that this job was initially rejected already at level 3 .

\section{Numerical experiments}

\subsection{The data}

To evaluate the performance of the different algorithms described in Section 4, we use the instances proposed in [8]. These instances are randomly generated within predefined intervals as follows:

- the processing times $p_{j}$ : random integer numbers within $[10,80]$,

- the weight values $w_{j}$ : random integer numbers within $[1,30]$,

- the rejection costs $r_{j}: \exp (5+\sqrt{a} b)$ where $a$ is a random integer number within $[1,80]$ and $b$ is a random number within $[0,1]$.

The number of jobs is fixed to 21 values:

- small instances with $n \in\{5,7,9,13,15,20\}$, 
- medium instances with $n \in\{25,30,35,40,45,50\}$,

- larger instances with $n \in\{60,70,80,90,100,125,150,175,200\}$.

Three instances are generated for each value of $n$ so that 63 instances are solved.

\subsection{The results}

The complete results are presented in Table 10, Table 12, Table 14 and Table 16 (see the Annex). In these tables we use the following notations.

$|P E(H)|$ the number of solutions obtained by algorithm $H$ and for the comparison between two algorithms $H$ and $\bar{H}$ :

- $C(H, \bar{H})=|\{x \in P E(H) \bigcap P E(\bar{H})\}|$ is the number of common solutions in $P E(H)$ and $P E(\bar{H})$,

- $D(H, \bar{H})=\mid\{x \in P E(H) \mid \exists y \in P E(\bar{H}), y$ dominating $x\} \mid$ is the number of solutions in $P E(H)$ dominated by a solution of $P E(\bar{H})$,

- $N D(H, \bar{H})=|P E(H)|-C(H, \bar{H})-D(H, \bar{H})$ is the number of solutions in $P E(H)$ not present in $P E(\bar{H})$ and not dominated by any solution of $P E(\bar{H})$.

One can find that, $\mathrm{H} 4$ is by so far the most performant algorithm: no of the solutions obtained by $\mathrm{H} 4$ is dominated by any solution generated with another algorithm $H$; moreover all the solutions generated by any $H$ are either found by $H 4$ or dominated by a solution of $H 4$. We have thus

$$
N D(H, H 4)=D(H 4, H)=0 \quad \forall H .
$$

For this reason the results of all the algorithms will be compared with those of $H 4$.

In Table 10 till Table 16, the first two columns characterize the instance:

(1) index of the 63 instances

(2) name of the instance $(n-x$, with $x \in\{1,2,3\})$

Table 10 is dedicated to the algorithm $H 1$ and its comparison with $H 4$. Columns 3 till 9, present the comparison of the results of $H 1 a$ and $H 1 b$ :

(3) $|P E(H 1 a)|$

(4) $D(H 1 a, H 1 b)$

(5) $N D(H 1 a, H 1 b)$

(6) $C(H 1 a, H 1 b)$

(7) $N D(H 1 b, H 1 a)$ 
(8) $D(H 1 b, H 1 a)$

(9) $|P E(H 1 b)|$

We have $(3)=(4)+(5)+(6)$ and $(9)=(6)+(7)+(8)$. Columns 10 to 12 present the comparison of the results of $H 1 a$ and $H 4$ :

(10) $D(H 1 a, H 4)$

(11) $C(H 1 a, H 4)$

(12) $N D(H 4, H 1 a)$

We have $|P E(H 1 a)|=(10)+(11),|P E(H 4)|=(11)+(12)($ as $N D(H 1 a, H 4)=0$ and $D(H 4, H 1 a)=0)$.

In the same way, columns 13 to 15 present the comparison of the results of $H 1 b$ and $H 4$ and columns 16 to 18 present the comparison of the results of $H 1 a b$ and $H 4$.

With the same structure of Table 10, Table 12 and Table 14 consider respectively the comparison of $H 2$ and $H 3$ with the algorithm $H 4$.

Table 16 analyses the performance of the different algorithms, indicating the ratio

$$
\%(H, H 4)=\frac{C(H, H 4)}{|P E(H 4)|}
$$

i.e. the percentage of the solutions of $H 4$ generated by algorithm $H$.

This percentage is given in columns 5, 7 and 9 for $H 1 a, H 1 b$ and $H 1 a b$, in columns 10, 12 and 14 for $H 2 a, H 2 b$ and $H 2 a b$, and in columns 15, 17 and 19 for $H 3 a, H 3 b$ and $H 3 a b$. Table 16 gives also the CPU time for the different algorithms, in columns 6 and 8 for $H 1 a$ and $H 1 b$, columns 11 and 13 for $H 2 a$ and $H 2 b$, columns 16 and 18 for $H 3 a$ and $H 3 b$. Column 3 gives the number of solutions obtained with algorithm $H 4$ and column 4 gives the CPU time for algorithm $H 4$. Only the CPU times greater or equal to 1 second are reported.

Let us note that the filtering process-time to obtain the result of an algorithm $\mathrm{Hab}$ is almost immediate.

Finally, Table 18 presents a more synthetic view of all these results, gathering the instances by three successive values of $n$. These values are indicated in column 1 and correspond to 9 instances. The nine other columns give the average value of $\%(H, H 4)$ in these instances, respectively for the 9 algorithms from $H 1 a$ till $H 3 a b$. The last line of Table 18 resumes all the results by the average value of $\%(H, H 4)$ for all the instances.

\section{Remark 3:}

In [8], all these instances are solved to also generate $P E$, with two metaheuristics: MOSA [10] and NSGAII [3]. Unfortunately, it appears impossible to compare our results with those of [8] for the following reasons:

- with NSGAII, the number of solutions in PE is always limited to 100, 
- for MOSA, the parameters are not well fixed because they do not dynamically vary in function of the dimension of the instances.

So in the results of [8] the average numbers of solutions in $P E$ for the instances with $n=200$, are respectively equal to 87 with MOSA and 100 with NSGAII, to compare with the thousands solutions in PE in the results of Table 10, Table 12 and Table 14.

\subsection{Analysis of the results}

\subsection{1. $H 3$ versus $H 4$}

First of all, Table 14 and Table 16 indicate that the two algorithms $H 3 a$ and especially $H 3 b$ give excellent results according to $H 4$, with very large ratios $\%(H 3 a, H 4)$ and $\%(H 3 b, H 4)$. But it is remarkable that $D(H 3 a b, H 4)=N D(H 4, H 3 a b)=0$ so that $\%(H 3 a b, H 4)=100$ for all the instances. $H 3 a b$ and $H 4$ produce thus the same sets $P E(H 3 a b)$ and $P E(H 4)$. Is it the exact set of efficient solutions ? It is an open question to further research. In any case, we take thus this set, denoted by $\hat{E}$, as a reference set to compare the different algorithms.

\subsubsection{The small-size instances $(n \leq 20)$}

For this set of instances, it has been possible to verify that $\hat{E}$ is the exact set of efficient solutions by a complete enumeration of all the feasible solutions.

For the 18 small instances, even if the results of the 3 algorithms $H 1 a, H 2 a$ and $H 3 a$ are quite good, nevertheless sometimes they miss some solutions of $\hat{E}$; it is the case for 5 instances (12-13-16-17-18) for $H 1 a$ and $H 3 a$ and for 3 instances (13-1618) with $H 2 a$ (see Table 16). But it is not the case for the versions $H 1 b, H 2 b$ and $H 3 b$ which generate exactly $\hat{E}$ (see Table 16 ). In these instances, it appears that the missing solutions of the versions $a$, are located at a high level of the branching scheme and for this reason, they are more easily obtained with the versions $b$.

\subsubsection{The medium-size instances $(25 \leq n \leq 50)$}

Often, the versions $b$ of the algorithms produce a larger percentage of $\hat{E}$ than the corresponding versions $a$, but it is not always the case. Effectively, in particular for $H 1$, we have opposite results: for example, with instance 27 , we have $\%(H 1 b, H 4)=98.9$ however only $\%(H 1 a, H 4)=54.1$ otherwise with the instance 36 the results are different, $\%(H 1 b, H 4)=42.0$ instead $\%(H 1 a, H 4)=95.4$. The respective performance of the versions $a$ and $b$ depends on the data of each instance. Nevertheless, for these medium-size instances, the results of $H 1 a b$ are excellent with an average percentage $\%(H 1 a b, H 4)$ larger than $99.83 \%$ (see Table 18 ).

It is even the case when either the result of $H 1 a$ is bad (see the instance 27 in 
Table 16 where $\%(H 1 a b, H 4)=99.3)$ or the result of $H 1 b$ is bad (see the instance 36 in Table $16 \%(H 1 a b, H 4)=99.0)$.

We can note that $H 2$ becomes a little bit more competitive than $H 1$ for these medium-size instances, and $H 3$ is more competitive than $H 2$.

The version $b$ of $H 2$ and $H 3$ are almost always more performant than their version $a$, with some rare exceptions like instances 27, 35 and 36 for $\mathrm{H} 2$ and instance 27 for $H 3$ (see Table 16$)$. But, as with $H 3$, we have always $\%(H 2 a b, H 4)=100$ so that for the medium instances $P E(H 2 a b)=\hat{E}$.

\subsubsection{The large-size instances $(60 \leq n \leq 200)$}

For the large instances, clearly algorithms $H 1 a$ and $H 1 b$ become inadequate to obtain a good approximation of $\hat{E}$, with small ratios $\%(H 1 a, H 4)$ and $\%(H 1 b, H 4)$. Table 10 shows also that the numbers $D(H 1 a, H 4)$ and $D(H 1 b, H 4)$ are very large, especially when $n \geq 150$ (with rare exception like $D(H 1 a, H 4)$ for instance 59 ); it appears that these algorithms produce thousand solutions which in fact are dominated.

It should be underlined that in many of these large instances, we have only $C(H 1 a, H 1 b)=2$ (see Table 10$)$. These two unique common solutions in PE $(H 1 a)$ and $P E(H 1 b)$ are in fact the two extreme efficient solutions corresponding to rejecting none of the jobs or rejecting all of them. Except these two obvious solutions, $H 1 a$ and $H 1 b$ completely diverge to generate $P E(H 1 a)$ and $P E(H 1 b)$.

Even if the results of $H 2$ improve those of $H 1$, we note (see Table 12) that $D(H 2 a, H 4)$ and $D(H 2 b, H 4)$ increase with the dimension of the instances to reach several thousands when $n \geq 150$. So these algorithms do not provide a good approximation of $\hat{E}$ for instances with very large dimension.

For such instances, there is a clear superiority of $H 3$ versus $H 2$. The performance of $H 3$ remains excellent and very stable with, in particular of $H 3 b$, a ratio \% $(H 3 b, H 4)$ larger than 99.1 (see Table 16). We recall that for all the instances $\%(H 3 a b, H 4)=$ 100 .

\subsubsection{The CPU time}

Concerning the CPU time of all the different algorithms, we can see from Table 16 (columns 4,6,8,11,13,16 and 18) that:

- for $H 1$ and $H 3$, it is always inferior to 2 seconds, even when $n=200$,

- logically, $H 2$ needs a larger CPU time till 35 seconds for $H 2 a$ and 16 seconds for $H 2 b$ when $n=200$,

- $H 4$ takes 19 seconds to treat the instances with $n=200$, thus a larger time than $H 3 a$ and $H 3 b$. We must note that the filtering process of their respective set $P E$ to obtain $P E(H 3 a b)$ is almost immediate.

In conclusion of this analysis, $H 4$ appears the more efficient and stable algorithm to approximate $E$ even if $H 3 a b$ appears as an excellent alternative, a little faster. 


\subsection{Comment on the implementation of the algorithms}

The algorithms presented in the paper are all implicit enumeration. At each iteration, a solution $s$ (related to a node of the tree) is selected from the pool and is examined by the branching scheme. The set $E(s)$ of children solutions of the parent solution $s$ is created with only solutions corresponding to a non-dominated variation. For the solution $s$, the variations corresponding to the two objectives $C_{w}$ and $R$ can be efficiently obtained using relation (9) and relation (10). Nevertheless relation (10) is computationally very efficient only when the vector of variations $\Delta^{()}$can also be stored in the pool. For information, to treat the instances with $n=200$, the maximal size of pool is equal to 1600 for $H 1, H 3$ and $H 4$, and is equal to 8000 for $H 2$. In case of limited memory (very large scale problems), we can proceed differently and these variations can still be calculated efficiently using relation (16) which is a generalization of relation (7).

$$
\Delta_{j}^{(k)}=p_{j}\left(\sum_{\substack{k \in \bar{A} \\ k \geq j}} w_{k}\right)+w_{j}\left(\sum_{\substack{l \in \bar{A} \\ l<j}} p_{l}\right)
$$

The verification of the non-dominance of the variations can be done efficiently when considering these variations in the order of increasing rejection cost. For this reason, an additional vector is created $\left(i_{1}, i_{2}, \ldots, i_{n}\right)$ corresponding to the increasing order $r_{i_{1}} \leq r_{i_{2}} \leq \ldots \leq r_{i_{n}}$. For the didactic example given in Table 1, we have that $r_{2} \leq r_{5} \leq r_{4} \leq r_{3} \leq r_{1}$ so that we create the additional vector $(2,5,4,3,1)$. We compare the successive variations $\left(r_{j_{k}}, \Delta_{j_{k}}^{()}\right)$and $\left(r_{j_{k}+1}, \Delta_{j_{k}+1}^{()}\right)$. These variations are non-dominated if $\Delta_{j_{k}}^{()}<\Delta_{j_{k}+1}^{()}$.

\section{Conclusion}

For the bi-objective model $1 / /\left(C_{w}, R\right)$ several heuristics which are implicit enumeration following a branching scheme are proposed. They all use the concept of nondominated variations to define the set $E(s)$ of children solutions of a parent solution $s$. These heuristics differ either by the definition of fathomed node or by the branching scheme. The large set of experimental results proofs that the heuristic $H 1$ and $H 2$ are not well adapted to large instances; secondly the superiority of the heuristic $H 4$, even if $H 3 a b$ obtains the same results.

For the future, one can first analyse if some metaheuristics are able to improve the results; of course to propose an exact method is also a challenge. It will also be interesting, in the spirit of [4], [6] and [8], to analyse if the concept of non-dominated variations can be extended to the Lorenz domination variations instead of the Pareto domination. Another direction of further research is to adapt these algorithms to other classical scheduling models, like two parallel machines or two sequential machines, with rejection. 


\section{References}

[1] Y. Bartal, S. Leonardi, A. Marchetti-Spaccamela, J. Sgall, L. Stougie, "Multiprocessor scheduling with rejection", Journal of Discrete Mathematics 13(1), 64-78, (2000).

[2] J. Blazewicz, K.H. Ecker, E. Pesch, G. Schmidt, J. Weglarz, "Handbook on scheduling : From theory to applications", Springer Berlin Heidelberg New York (2007).

[3] K. Deb, S. S. Agrawal, A. Pratap, T. Meyarivan, "A fast elitist non-dominated sorting genetic algorithm for multi-objective optimization: NSGA-II", Lecture Notes in Computer Sciences, 1917, 849-858, (2000).

[4] F. Dugardin, F. Yalaoui, L. Amodeo "New multi-objective method to solve reentrant hybrid flow shop scheduling problem", European Journal of Operational Research 203(1), 22-31, (2010).

[5] Z.-H. Jia, M.-L. Pei, J.Y.-T. Leung "Multi-objective ACO algorithms to minimize the makespan and the total rejection cost on BPMs with arbitrary job weights", International Journal of Systems Science 48 (16), 3542-3557, (2017).

[6] A. Moghaddam, F. Yalaoui, L. Amodeo, "Lorenz versus Pareto dominance in a single machine scheduling problem with rejection", Lecture Notes in Computer Science 6576, 520-534, (2011).

[7] A. Moghaddam, L. Amodeo, F. Yalaoui, B. Karimi "Single machine scheduling with rejection minimizing total weighted completion time and rejection cost", International Journal of Applied Evolutionary Computation 3 (2), 42-61, (2012).

[8] A. Moghaddam, F. Yalaoui, L. Amodeo, "Efficient meta-heuristics based on various dominance criteria for a single-machine bi-criteria scheduling problem with rejection", Journal of Manufacturing Systems 34(C), 12-22, (2015).

[9] V. T'Kindt, J.-Ch. Billaut, "Multicriteria scheduling. Theory, Models and Algorithms", Springer-Verlag Berlin Heidelberg New York (2002).

[10] E.L. Ulungu, J. Teghem, Ph. Fortemps, D. Tuyttens, "MOSA method : A tool for solving multiobjective combinatorial optimization problems", Journal of MultiCriteria Decision Analysis 8(4), 221-236, (1999).

[11] D.-J. Wang, Y. Yin, M. Liu, "Bicriteria scheduling problems involving job rejection, controllable processing times and rate-modifying activity", International Journal of Production Research 54(12), 3691-3705, (2016).

[12] L. Zhang, L. Lu, S. Li, "New results on two-machine flow-shop scheduling with rejection", Journal of Combinatorial Optimization 31 (4), 1493-1504, (2016).

Received 4.10.2018, Accepted 13.11.2018 
Annex: Instance data and complete results

Table 8: Data of instance 12

\begin{tabular}{c|ccc}
$j$ & $p_{j}$ & $w_{j}$ & $r_{j}$ \\
\hline 1 & 18 & 22 & 1602 \\
2 & 14 & 16 & 592 \\
3 & 26 & 18 & 15738 \\
4 & 40 & 21 & 432 \\
5 & 51 & 26 & 408 \\
6 & 23 & 8 & 601 \\
7 & 76 & 26 & 149 \\
8 & 80 & 27 & 809 \\
9 & 16 & 4 & 4017 \\
10 & 69 & 15 & 5879 \\
11 & 74 & 16 & 1053 \\
12 & 41 & 4 & 282 \\
13 & 63 & 4 & 1652
\end{tabular}

Table 9: Data of instance 13

\begin{tabular}{c|ccc}
$j$ & $p_{j}$ & $w_{j}$ & $r_{j}$ \\
\hline 1 & 11 & 25 & 9097 \\
2 & 26 & 29 & 39771 \\
3 & 53 & 28 & 1960 \\
4 & 73 & 27 & 2372 \\
5 & 44 & 13 & 11277 \\
6 & 22 & 6 & 20548 \\
7 & 42 & 11 & 2476 \\
8 & 75 & 19 & 1838 \\
9 & 77 & 13 & 427 \\
10 & 62 & 9 & 17825 \\
11 & 53 & 7 & 2273 \\
12 & 41 & 5 & 383 \\
13 & 66 & 6 & 2227 \\
14 & 64 & 2 & 175 \\
15 & 68 & 1 & 3782
\end{tabular}


Table 10: Results $H 1$ (Part I)

\begin{tabular}{|c|c|c|c|c|c|c|c|c|}
\hline (1) & $(2)$ & $(3)$ & $(4)$ & $(5)$ & (6) & $(7)$ & $(8)$ & $(9)$ \\
\hline & $n$ & $|P E(H 1 a)|$ & $\mathrm{D}$ & ND & $C(H 1 a, H 1 b)$ & ND & $\mathrm{D}$ & $P E(H 1 b)$ \\
\hline 1 & $5-1$ & 11 & 0 & 0 & 11 & 0 & 0 & 11 \\
\hline 2 & $5-2$ & 8 & 0 & 0 & 8 & 0 & 0 & 8 \\
\hline 3 & $5-3$ & 7 & 0 & 0 & 7 & 0 & 0 & 7 \\
\hline 4 & $7-1$ & 20 & 0 & 0 & 20 & 0 & 0 & 20 \\
\hline 5 & $7-2$ & 17 & 0 & 0 & 17 & 0 & 0 & 17 \\
\hline 6 & $7-3$ & 16 & 0 & 0 & 16 & 0 & 0 & 16 \\
\hline 7 & $9-1$ & 22 & 0 & 0 & 22 & 0 & 0 & 22 \\
\hline 8 & $9-2$ & 26 & 0 & 0 & 26 & 0 & 0 & 26 \\
\hline 9 & $9-3$ & 26 & 0 & 0 & 26 & 0 & 0 & 26 \\
\hline 10 & $13-1$ & 30 & 0 & 0 & 30 & 0 & 0 & 30 \\
\hline 11 & $13-2$ & 41 & 0 & 0 & 41 & 0 & 0 & 41 \\
\hline 12 & $13-3$ & 39 & 0 & 0 & 39 & 2 & 0 & 41 \\
\hline 13 & $15-1$ & 65 & 0 & 0 & 65 & 1 & 0 & 66 \\
\hline 14 & $15-2$ & 39 & 0 & 0 & 39 & 0 & 0 & 39 \\
\hline 15 & $15-3$ & 81 & 0 & 0 & 81 & 0 & 0 & 81 \\
\hline 16 & $20-1$ & 124 & 1 & 0 & 123 & 3 & 0 & 126 \\
\hline 17 & $20-2$ & 93 & 0 & 0 & 93 & 1 & 0 & 94 \\
\hline 18 & $20-3$ & 96 & 21 & 0 & 75 & 53 & 0 & 128 \\
\hline 19 & 25-1 & 144 & 0 & 0 & 144 & 5 & 0 & 149 \\
\hline 20 & $25-2$ & 108 & 0 & 0 & 108 & 6 & 0 & 114 \\
\hline 21 & $25-3$ & 156 & 0 & 3 & 153 & 3 & 2 & 158 \\
\hline 22 & $30-1$ & 218 & 0 & 6 & 212 & 5 & 1 & 218 \\
\hline 23 & $30-2$ & 133 & 1 & 0 & 132 & 7 & 0 & 139 \\
\hline 24 & $30-3$ & 221 & 0 & 0 & 221 & 7 & 0 & 228 \\
\hline 25 & $35-1$ & 288 & 0 & 24 & 264 & 17 & 14 & 295 \\
\hline 26 & $35-2$ & 242 & 1 & 0 & 241 & 33 & 0 & 274 \\
\hline 27 & $35-3$ & 192 & 39 & 1 & 152 & 128 & 0 & 280 \\
\hline 28 & $40-1$ & 298 & 0 & 0 & 298 & 7 & 0 & 305 \\
\hline 29 & $40-2$ & 292 & 0 & 74 & 218 & 12 & 49 & 279 \\
\hline 30 & $40-3$ & 296 & 0 & 0 & 296 & 18 & 0 & 314 \\
\hline 31 & $45-1$ & 290 & 0 & 2 & 288 & 6 & 0 & 294 \\
\hline 32 & $45-2$ & 352 & 0 & 79 & 273 & 7 & 47 & 327 \\
\hline 33 & $45-3$ & 348 & 0 & 54 & 294 & 12 & 36 & 342 \\
\hline 34 & $50-1$ & 374 & 2 & 123 & 249 & 4 & 107 & 360 \\
\hline 35 & $50-2$ & 401 & 2 & 177 & 222 & 12 & 156 & 390 \\
\hline 36 & $50-3$ & 559 & 0 & 334 & 225 & 21 & 290 & 536 \\
\hline 37 & $60-1$ & 526 & 1 & 148 & 377 & 6 & 56 & 439 \\
\hline 38 & $60-2$ & 481 & 317 & 0 & 164 & 335 & 0 & 499 \\
\hline 39 & $60-3$ & 485 & 14 & 0 & 471 & 76 & 0 & 547 \\
\hline 40 & $70-1$ & 622 & 5 & 10 & 607 & 72 & 0 & 679 \\
\hline 41 & $70-2$ & 708 & 621 & 85 & 2 & 677 & 97 & 776 \\
\hline 42 & $70-3$ & 776 & 69 & 602 & 105 & 76 & 495 & 676 \\
\hline 43 & $80-1$ & 983 & 903 & 0 & 80 & 926 & 0 & 1006 \\
\hline 44 & $80-2$ & 676 & 1 & 531 & 144 & 6 & 506 & 656 \\
\hline 45 & $80-3$ & 839 & 22 & 622 & 195 & 98 & 535 & 828 \\
\hline 46 & $90-1$ & 970 & 542 & 426 & 2 & 558 & 406 & 966 \\
\hline 47 & $90-2$ & 854 & 741 & 93 & 20 & 869 & 94 & 983 \\
\hline 48 & $90-3$ & 1083 & 265 & 647 & 171 & 445 & 638 & 1254 \\
\hline 49 & 100-1 & 1400 & 18 & 241 & 1141 & 47 & 127 & 1315 \\
\hline 50 & $100-2$ & 1037 & 412 & 623 & 2 & 440 & 613 & 1055 \\
\hline 51 & $100-3$ & 1190 & 21 & 641 & 528 & 60 & 512 & 1100 \\
\hline 52 & 125-1 & 1597 & 17 & 981 & 599 & 76 & 790 & 1465 \\
\hline 53 & $125-2$ & 1687 & 1401 & 284 & 2 & 1601 & 347 & 1950 \\
\hline 54 & 125-3 & 1691 & 3 & 1128 & 560 & 82 & 1072 & 1714 \\
\hline 55 & 150-1 & 3245 & 1071 & 2172 & 2 & 784 & 1670 & 2456 \\
\hline 56 & 150-2 & 2262 & 306 & 1954 & 2 & 589 & 2074 & 2665 \\
\hline 57 & $150-3$ & 2322 & 1615 & 705 & 2 & 1988 & 714 & 2704 \\
\hline 58 & 175-1 & 3054 & 1477 & 1575 & 2 & 1484 & 1571 & 3057 \\
\hline 59 & $175-2$ & 3317 & 2 & 3198 & 117 & 16 & 3011 & 3144 \\
\hline 60 & $175-3$ & 3377 & 1032 & 2343 & 2 & 1103 & 2273 & 3378 \\
\hline 61 & 200-1 & 3608 & 1762 & 1844 & 2 & 1833 & 1961 & 3796 \\
\hline 62 & $200-2$ & 3860 & 2488 & 1370 & 2 & 2775 & 1361 & 4138 \\
\hline 63 & $200-3$ & 3888 & 1924 & 1962 & 2 & 2210 & 1949 & 4161 \\
\hline
\end{tabular}


Table 11: Results $H 1$ (Part II)

\begin{tabular}{|c|c|c|c|c|c|c|c|c|}
\hline$(10)$ & $(11)$ & $(12)$ & $(13)$ & $(14)$ & $(15)$ & $(16)$ & $(17)$ & $(18)$ \\
\hline $\mathrm{D}$ & $C(H 1 a, H 4)$ & ND & $\mathrm{D}$ & $C(H 1 b, H 4)$ & ND & $\mathrm{D}$ & $C(H 1 a b, H 4)$ & $\mathrm{ND}$ \\
\hline 0 & 11 & 0 & 0 & 11 & 0 & 0 & 11 & 0 \\
\hline 0 & 8 & 0 & 0 & 8 & 0 & 0 & 8 & 0 \\
\hline 0 & 7 & 0 & 0 & 7 & 0 & 0 & 7 & 0 \\
\hline 0 & 20 & 0 & 0 & 20 & 0 & 0 & 20 & 0 \\
\hline 0 & 17 & 0 & 0 & 17 & 0 & 0 & 17 & 0 \\
\hline 0 & 16 & 0 & 0 & 16 & 0 & 0 & 16 & 0 \\
\hline 0 & 22 & 0 & 0 & 22 & 0 & 0 & 22 & 0 \\
\hline 0 & 26 & 0 & 0 & 26 & 0 & 0 & 26 & 0 \\
\hline 0 & 26 & 0 & 0 & 26 & 0 & 0 & 26 & 0 \\
\hline 0 & 30 & 0 & 0 & 30 & 0 & 0 & 30 & 0 \\
\hline 0 & 41 & 0 & 0 & 41 & 0 & 0 & 41 & 0 \\
\hline 0 & 39 & 2 & 0 & 41 & 0 & 0 & 41 & 0 \\
\hline 0 & 65 & 1 & 0 & 66 & 0 & 0 & 66 & 0 \\
\hline 0 & 39 & 0 & 0 & 39 & 0 & 0 & 39 & 0 \\
\hline 0 & 81 & 0 & 0 & 81 & 0 & 0 & 81 & 0 \\
\hline 1 & 123 & 3 & 0 & 126 & 0 & 0 & 126 & 0 \\
\hline 0 & 93 & 1 & 0 & 94 & 0 & 0 & 94 & 0 \\
\hline 21 & 75 & 53 & 0 & 128 & 0 & 0 & 128 & 0 \\
\hline 0 & 144 & 5 & 0 & 149 & 0 & 0 & 149 & 0 \\
\hline 0 & 108 & 6 & 0 & 114 & 0 & 0 & 114 & 0 \\
\hline 0 & 156 & 3 & 2 & 156 & 3 & 0 & 159 & 0 \\
\hline 0 & 218 & 5 & 1 & 217 & 6 & 0 & 223 & 0 \\
\hline 1 & 132 & 7 & 0 & 139 & 0 & 0 & 139 & 0 \\
\hline 0 & 221 & 7 & 0 & 228 & 0 & 0 & 228 & 0 \\
\hline 0 & 288 & 17 & 14 & 281 & 24 & 0 & 305 & 0 \\
\hline 1 & 241 & 33 & 0 & 274 & 0 & 0 & 274 & 0 \\
\hline 39 & 153 & 130 & 0 & 280 & 3 & 0 & 281 & 2 \\
\hline 0 & 298 & 7 & 0 & 305 & 0 & 0 & 305 & 0 \\
\hline 0 & 292 & 12 & 49 & 230 & 74 & 0 & 304 & 0 \\
\hline 0 & 296 & 18 & 0 & 314 & 0 & 0 & 314 & 0 \\
\hline 0 & 290 & 6 & 0 & 294 & 2 & 0 & 296 & 0 \\
\hline 0 & 352 & 7 & 47 & 280 & 79 & 0 & 359 & 0 \\
\hline 0 & 348 & 12 & 36 & 306 & 54 & 0 & 360 & 0 \\
\hline 2 & 372 & 5 & 107 & 253 & 124 & 0 & 376 & 1 \\
\hline 3 & 398 & 13 & 156 & 234 & 177 & 1 & 410 & 1 \\
\hline 0 & 559 & 27 & 290 & 246 & 340 & 0 & 580 & 6 \\
\hline 1 & 525 & 9 & 56 & 383 & 151 & 0 & 531 & 3 \\
\hline 317 & 164 & 341 & 2 & 497 & 8 & 2 & 497 & 8 \\
\hline 14 & 471 & 76 & 0 & 547 & 0 & 0 & 547 & 0 \\
\hline 5 & 617 & 72 & 0 & 679 & 10 & 0 & 689 & 0 \\
\hline 638 & 70 & 760 & 162 & 614 & 216 & 82 & 682 & 148 \\
\hline 72 & 704 & 86 & 495 & 181 & 609 & 3 & 780 & 10 \\
\hline 903 & 80 & 930 & 2 & 1004 & 6 & 2 & 1004 & 6 \\
\hline 1 & 675 & 13 & 507 & 149 & 539 & 1 & 680 & 8 \\
\hline 25 & 814 & 125 & 535 & 293 & 646 & 3 & 912 & 27 \\
\hline 727 & 243 & 783 & 484 & 482 & 544 & 263 & 723 & 303 \\
\hline 764 & 90 & 899 & 210 & 773 & 216 & 121 & 861 & 128 \\
\hline 374 & 709 & 669 & 740 & 514 & 864 & 190 & 1073 & 305 \\
\hline 18 & 1382 & 53 & 127 & 1188 & 247 & 0 & 1429 & 6 \\
\hline 875 & 162 & 1010 & 765 & 290 & 882 & 615 & 450 & 722 \\
\hline 23 & 1167 & 78 & 512 & 588 & 657 & 2 & 1227 & 18 \\
\hline 25 & 1572 & 125 & 806 & 659 & 1038 & 20 & 1636 & 61 \\
\hline 1529 & 158 & 1922 & 993 & 957 & 1123 & 774 & 1113 & 967 \\
\hline 38 & 1653 & 128 & 1078 & 636 & 1145 & 35 & 1735 & 46 \\
\hline 2021 & 1224 & 1587 & 1943 & 513 & 2298 & 1223 & 1735 & 1076 \\
\hline 1756 & 506 & 2582 & 2335 & 330 & 2758 & 1711 & 834 & 2254 \\
\hline 1762 & 560 & 2832 & 1407 & 1297 & 2095 & 840 & 1855 & 1537 \\
\hline 2556 & 498 & 2860 & 2329 & 728 & 2630 & 1837 & 1224 & 2134 \\
\hline 133 & 3184 & 314 & 3011 & 133 & 3365 & 131 & 3200 & 298 \\
\hline 3238 & 139 & 3474 & 2962 & 416 & 3197 & 2895 & 553 & 3060 \\
\hline 3116 & 492 & 3340 & 2738 & 1058 & 2774 & 2131 & 1548 & 2284 \\
\hline 3500 & 360 & 3914 & 2577 & 1561 & 2713 & 2228 & 1919 & 2355 \\
\hline 3341 & 547 & 3877 & 3324 & 837 & 3587 & 2792 & 1382 & 3042 \\
\hline
\end{tabular}


Table 12: Results H2 (Part I)

\begin{tabular}{|c|c|c|c|c|c|c|c|c|}
\hline (1) & $(2)$ & $(3)$ & $(4)$ & $(5)$ & (6) & $(7)$ & $(8)$ & $(9)$ \\
\hline & $n$ & $|P E(H 2 a)|$ & $\mathrm{D}$ & ND & $C(H 2 a, H 2 b)$ & ND & $\mathrm{D}$ & $|P E(H 2 b)|$ \\
\hline 1 & $5-1$ & 11 & 0 & 0 & 11 & 0 & 0 & 11 \\
\hline 2 & $5-2$ & 8 & 0 & 0 & 8 & 0 & 0 & 8 \\
\hline 3 & $5-3$ & 7 & 0 & 0 & 7 & 0 & 0 & 7 \\
\hline 4 & $7-1$ & 20 & 0 & 0 & 20 & 0 & 0 & 20 \\
\hline 5 & $7-2$ & 17 & 0 & 0 & 17 & 0 & 0 & 17 \\
\hline 6 & $7-3$ & 16 & 0 & 0 & 16 & 0 & 0 & 16 \\
\hline 7 & $9-1$ & 22 & 0 & 0 & 22 & 0 & 0 & 22 \\
\hline 8 & $9-2$ & 26 & 0 & 0 & 26 & 0 & 0 & 26 \\
\hline 9 & $9-3$ & 26 & 0 & 0 & 26 & 0 & 0 & 26 \\
\hline 10 & $13-1$ & 30 & 0 & 0 & 30 & 0 & 0 & 30 \\
\hline 11 & $13-2$ & 41 & 0 & 0 & 41 & 0 & 0 & 41 \\
\hline 12 & $13-3$ & 41 & 0 & 0 & 41 & 0 & 0 & 41 \\
\hline 13 & $15-1$ & 65 & 0 & 0 & 65 & 1 & 0 & 66 \\
\hline 14 & $15-2$ & 39 & 0 & 0 & 39 & 0 & 0 & 39 \\
\hline 15 & $15-3$ & 81 & 0 & 0 & 81 & 0 & 0 & 81 \\
\hline 16 & 20-1 & 124 & 1 & 0 & 123 & 3 & 0 & 126 \\
\hline 17 & $20-2$ & 94 & 0 & 0 & 94 & 0 & 0 & 94 \\
\hline 18 & $20-3$ & 123 & 0 & 0 & 123 & 5 & 0 & 128 \\
\hline 19 & $25-1$ & 144 & 0 & 0 & 144 & 5 & 0 & 149 \\
\hline 20 & $25-2$ & 114 & 0 & 0 & 114 & 0 & 0 & 114 \\
\hline 21 & $25-3$ & 158 & 1 & 0 & 157 & 2 & 0 & 159 \\
\hline 22 & 30-1 & 218 & 0 & 3 & 215 & 5 & 1 & 221 \\
\hline 23 & $30-2$ & 136 & 2 & 0 & 134 & 5 & 0 & 139 \\
\hline 24 & $30-3$ & 226 & 0 & 0 & 226 & 2 & 0 & 228 \\
\hline 25 & $35-1$ & 300 & 0 & 2 & 298 & 5 & 1 & 304 \\
\hline 26 & $35-2$ & 258 & 0 & 0 & 258 & 16 & 0 & 274 \\
\hline 27 & $35-3$ & 220 & 7 & 0 & 213 & 70 & 0 & 283 \\
\hline 28 & $40-1$ & 303 & 0 & 0 & 303 & 2 & 0 & 305 \\
\hline 29 & $40-2$ & 293 & 0 & 0 & 293 & 11 & 0 & 304 \\
\hline 30 & $40-3$ & 298 & 0 & 0 & 298 & 16 & 0 & 314 \\
\hline 31 & $45-1$ & 293 & 0 & 2 & 291 & 3 & 0 & 294 \\
\hline 32 & $45-2$ & 355 & 1 & 5 & 349 & 5 & 0 & 354 \\
\hline 33 & $45-3$ & 353 & 1 & 0 & 352 & 8 & 0 & 360 \\
\hline 34 & $50-1$ & 374 & 2 & 9 & 363 & 5 & 2 & 370 \\
\hline 35 & $50-2$ & 405 & 3 & 37 & 365 & 9 & 24 & 398 \\
\hline 36 & $50-3$ & 565 & 2 & 82 & 481 & 23 & 46 & 550 \\
\hline 37 & 60-1 & 527 & 1 & 3 & 523 & 8 & 0 & 531 \\
\hline 38 & $60-2$ & 485 & 68 & 0 & 417 & 88 & 0 & 505 \\
\hline 39 & $60-3$ & 516 & 12 & 0 & 504 & 43 & 0 & 547 \\
\hline 40 & $70-1$ & 622 & 5 & 7 & 610 & 72 & 0 & 682 \\
\hline 41 & $70-2$ & 786 & 14 & 69 & 703 & 53 & 37 & 793 \\
\hline 42 & $70-3$ & 775 & 43 & 24 & 708 & 57 & 9 & 774 \\
\hline 43 & $80-1$ & 768 & 333 & 2 & 433 & 574 & 0 & 1007 \\
\hline 44 & $80-2$ & 678 & 1 & 3 & 674 & 11 & 3 & 688 \\
\hline 45 & $80-3$ & 845 & 23 & 296 & 526 & 108 & 217 & 851 \\
\hline 46 & $90-1$ & 964 & 475 & 84 & 405 & 494 & 53 & 952 \\
\hline 47 & $90-2$ & 900 & 49 & 7 & 844 & 144 & 4 & 992 \\
\hline 48 & $90-3$ & 1089 & 323 & 2 & 764 & 613 & 0 & 1377 \\
\hline 49 & 100-1 & 1410 & 13 & 438 & 959 & 32 & 378 & 1369 \\
\hline 50 & $100-2$ & 1056 & 63 & 34 & 959 & 176 & 10 & 1145 \\
\hline 51 & $100-3$ & 1203 & 4 & 20 & 1179 & 42 & 5 & 1226 \\
\hline 52 & 125-1 & 1632 & 14 & 389 & 1229 & 51 & 354 & 1634 \\
\hline 53 & $125-2$ & 1415 & 937 & 0 & 478 & 1572 & 0 & 2050 \\
\hline 54 & 125-3 & 1730 & 10 & 665 & 1055 & 58 & 621 & 1734 \\
\hline 55 & 150-1 & 2631 & 715 & 889 & 1027 & 722 & 725 & 2474 \\
\hline 56 & 150-2 & 2209 & 630 & 1176 & 403 & 1235 & 1197 & 2835 \\
\hline 57 & $150-3$ & 2222 & 1379 & 698 & 145 & 1858 & 707 & 2710 \\
\hline 58 & 175-1 & 2833 & 2044 & 123 & 666 & 2344 & 93 & 3103 \\
\hline 59 & $175-2$ & 3317 & 29 & 1934 & 1354 & 92 & 1861 & 3307 \\
\hline 60 & $175-3$ & 3173 & 2700 & 244 & 229 & 2950 & 213 & 3392 \\
\hline 61 & 200-1 & 3566 & 1986 & 1466 & 114 & 2116 & 1421 & 3651 \\
\hline 62 & $200-2$ & 3778 & 2675 & 690 & 413 & 3092 & 638 & 4143 \\
\hline 63 & $200-3$ & 3443 & 2319 & 8 & 1116 & 3253 & 3 & 4372 \\
\hline
\end{tabular}


Table 13: Results H2 (Part II)

\begin{tabular}{|c|c|c|c|c|c|c|c|c|}
\hline$(10)$ & (11) & $(12)$ & (13) & (14) & $(15)$ & $(16)$ & $(17)$ & $(18)$ \\
\hline $\mathrm{D}$ & $C(H 2 a, H 4)$ & ND & $\mathrm{D}$ & $C(H 2 b, H 4)$ & ND & $\mathrm{D}$ & $C(H 2 a b, H 4)$ & ND \\
\hline 0 & 11 & 0 & 0 & 11 & 0 & 0 & 11 & 0 \\
\hline 0 & 8 & 0 & 0 & 8 & 0 & 0 & 8 & 0 \\
\hline 0 & 7 & 0 & 0 & 7 & 0 & 0 & 7 & 0 \\
\hline 0 & 20 & 0 & 0 & 20 & 0 & 0 & 20 & 0 \\
\hline 0 & 17 & 0 & 0 & 17 & 0 & 0 & 17 & 0 \\
\hline 0 & 16 & 0 & 0 & 16 & 0 & 0 & 16 & 0 \\
\hline 0 & 22 & 0 & 0 & 22 & 0 & 0 & 22 & 0 \\
\hline 0 & 26 & 0 & 0 & 26 & 0 & 0 & 26 & 0 \\
\hline 0 & 26 & 0 & 0 & 26 & 0 & 0 & 26 & 0 \\
\hline 0 & 30 & 0 & 0 & 30 & 0 & 0 & 30 & 0 \\
\hline 0 & 41 & 0 & 0 & 41 & 0 & 0 & 41 & 0 \\
\hline 0 & 41 & 0 & 0 & 41 & 0 & 0 & 41 & 0 \\
\hline 0 & 65 & 1 & 0 & 66 & 0 & 0 & 66 & 0 \\
\hline 0 & 39 & 0 & 0 & 39 & 0 & 0 & 39 & 0 \\
\hline 0 & 81 & 0 & 0 & 81 & 0 & 0 & 81 & 0 \\
\hline 1 & 123 & 3 & 0 & 126 & 0 & 0 & 126 & 0 \\
\hline 0 & 94 & 0 & 0 & 94 & 0 & 0 & 94 & 0 \\
\hline 0 & 123 & 5 & 0 & 128 & 0 & 0 & 128 & 0 \\
\hline 0 & 144 & 5 & 0 & 149 & 0 & 0 & 149 & 0 \\
\hline 0 & 114 & 0 & 0 & 114 & 0 & 0 & 114 & 0 \\
\hline 1 & 157 & 2 & 0 & 159 & 0 & 0 & 159 & 0 \\
\hline 0 & 218 & 5 & 1 & 220 & 3 & 0 & 223 & 0 \\
\hline 2 & 134 & 5 & 0 & 139 & 0 & 0 & 139 & 0 \\
\hline 0 & 226 & 2 & 0 & 228 & 0 & 0 & 228 & 0 \\
\hline 0 & 300 & 5 & 1 & 303 & 2 & 0 & 305 & 0 \\
\hline 0 & 258 & 16 & 0 & 274 & 0 & 0 & 274 & 0 \\
\hline 7 & 213 & 70 & 0 & 283 & 0 & 0 & 283 & 0 \\
\hline 0 & 303 & 2 & 0 & 305 & 0 & 0 & 305 & 0 \\
\hline 0 & 293 & 11 & 0 & 304 & 0 & 0 & 304 & 0 \\
\hline 0 & 298 & 16 & 0 & 314 & 0 & 0 & 314 & 0 \\
\hline 0 & 293 & 3 & 0 & 294 & 2 & 0 & 296 & 0 \\
\hline 1 & 354 & 5 & 0 & 354 & 5 & 0 & 359 & 0 \\
\hline 1 & 352 & 8 & 0 & 360 & 0 & 0 & 360 & 0 \\
\hline 2 & 372 & 5 & 2 & 368 & 9 & 0 & 377 & 0 \\
\hline 3 & 402 & 9 & 24 & 374 & 37 & 0 & 411 & 0 \\
\hline 2 & 563 & 23 & 46 & 504 & 82 & 0 & 586 & 0 \\
\hline 1 & 526 & 8 & 0 & 531 & 3 & 0 & 534 & 0 \\
\hline 68 & 417 & 88 & 0 & 505 & 0 & 0 & 505 & 0 \\
\hline 12 & 504 & 43 & 0 & 547 & 0 & 0 & 547 & 0 \\
\hline 5 & 617 & 72 & 0 & 682 & 7 & 0 & 689 & 0 \\
\hline 16 & 770 & 60 & 41 & 752 & 78 & 4 & 821 & 9 \\
\hline 43 & 732 & 58 & 9 & 765 & 25 & 0 & 789 & 1 \\
\hline 333 & 435 & 575 & 0 & 1007 & 3 & 0 & 1009 & 1 \\
\hline 1 & 677 & 11 & 3 & 685 & 3 & 0 & 688 & 0 \\
\hline 25 & 820 & 119 & 217 & 634 & 305 & 2 & 928 & 11 \\
\hline 546 & 418 & 608 & 136 & 816 & 210 & 154 & 829 & 197 \\
\hline 105 & 795 & 194 & 90 & 902 & 87 & 86 & 909 & 80 \\
\hline 325 & 764 & 614 & 5 & 1372 & 6 & 5 & 1374 & 4 \\
\hline 17 & 1393 & 42 & 380 & 989 & 446 & 4 & 1425 & 10 \\
\hline 63 & 993 & 179 & 11 & 1134 & 38 & 1 & 1168 & 4 \\
\hline 5 & 1198 & 47 & 5 & 1221 & 24 & 1 & 1240 & 5 \\
\hline 18 & 1614 & 83 & 354 & 1280 & 417 & 4 & 1665 & 32 \\
\hline 938 & 477 & 1603 & 35 & 2015 & 65 & 35 & 2015 & 65 \\
\hline 38 & 1692 & 89 & 625 & 1109 & 672 & 28 & 1750 & 31 \\
\hline 784 & 1847 & 964 & 769 & 1705 & 1106 & 83 & 2555 & 256 \\
\hline 1367 & 842 & 2246 & 1963 & 872 & 2216 & 1499 & 1315 & 1773 \\
\hline 1528 & 694 & 2698 & 1403 & 1307 & 2085 & 843 & 1858 & 1534 \\
\hline 2198 & 635 & 2723 & 861 & 2242 & 1116 & 888 & 2245 & 1113 \\
\hline 133 & 3184 & 314 & 1868 & 1439 & 2059 & 106 & 3274 & 224 \\
\hline 2887 & 286 & 3327 & 887 & 2505 & 1108 & 861 & 2562 & 1051 \\
\hline 3062 & 504 & 3328 & 2409 & 1242 & 2590 & 2064 & 1632 & 2200 \\
\hline 3362 & 416 & 3858 & 1920 & 2223 & 2051 & 1969 & 2226 & 2048 \\
\hline 2322 & 1121 & 3303 & 61 & 4311 & 113 & 58 & 4319 & 105 \\
\hline
\end{tabular}


Table 14: Results H3 (Part I)

\begin{tabular}{|c|c|c|c|c|c|c|c|c|}
\hline (1) & $(2)$ & $(3)$ & $(4)$ & $(5)$ & (6) & $(7)$ & $(8)$ & $(9)$ \\
\hline & $n$ & $|P E(H 3 a)|$ & $\mathrm{D}$ & ND & $C(H 3 a, H 3 b)$ & ND & $\mathrm{D}$ & $P E(H 3 b)$ \\
\hline 1 & $5-1$ & 11 & 0 & 0 & 11 & 0 & 0 & 11 \\
\hline 2 & $5-2$ & 8 & 0 & 0 & 8 & 0 & 0 & 8 \\
\hline 3 & $5-3$ & 7 & 0 & 0 & 7 & 0 & 0 & 7 \\
\hline 4 & $7-1$ & 20 & 0 & 0 & 20 & 0 & 0 & 20 \\
\hline 5 & $7-2$ & 17 & 0 & 0 & 17 & 0 & 0 & 17 \\
\hline 6 & $7-3$ & 16 & 0 & 0 & 16 & 0 & 0 & 16 \\
\hline 7 & $9-1$ & 22 & 0 & 0 & 22 & 0 & 0 & 22 \\
\hline 8 & $9-2$ & 26 & 0 & 0 & 26 & 0 & 0 & 26 \\
\hline 9 & $9-3$ & 26 & 0 & 0 & 26 & 0 & 0 & 26 \\
\hline 10 & $13-1$ & 30 & 0 & 0 & 30 & 0 & 0 & 30 \\
\hline 11 & $13-2$ & 41 & 0 & 0 & 41 & 0 & 0 & 41 \\
\hline 12 & $13-3$ & 40 & 0 & 0 & 40 & 1 & 0 & 41 \\
\hline 13 & $15-1$ & 65 & 0 & 0 & 65 & 1 & 0 & 66 \\
\hline 14 & $15-2$ & 39 & 0 & 0 & 39 & 0 & 0 & 39 \\
\hline 15 & $15-3$ & 81 & 0 & 0 & 81 & 0 & 0 & 81 \\
\hline 16 & $20-1$ & 124 & 1 & 0 & 123 & 3 & 0 & 126 \\
\hline 17 & $20-2$ & 93 & 0 & 0 & 93 & 1 & 0 & 94 \\
\hline 18 & $20-3$ & 122 & 1 & 0 & 121 & 7 & 0 & 128 \\
\hline 19 & $25-1$ & 144 & 0 & 0 & 144 & 5 & 0 & 149 \\
\hline 20 & $25-2$ & 108 & 0 & 0 & 108 & 6 & 0 & 114 \\
\hline 21 & $25-3$ & 157 & 0 & 0 & 157 & 2 & 0 & 159 \\
\hline 22 & $30-1$ & 219 & 0 & 0 & 219 & 4 & 0 & 223 \\
\hline 23 & $30-2$ & 138 & 0 & 0 & 138 & 1 & 0 & 139 \\
\hline 24 & $30-3$ & 221 & 0 & 0 & 221 & 7 & 0 & 228 \\
\hline 25 & $35-1$ & 289 & 0 & 0 & 289 & 16 & 0 & 305 \\
\hline 26 & $35-2$ & 274 & 0 & 0 & 274 & 0 & 0 & 274 \\
\hline 27 & $35-3$ & 282 & 0 & 3 & 279 & 1 & 0 & 280 \\
\hline 28 & $40-1$ & 301 & 0 & 0 & 301 & 4 & 0 & 305 \\
\hline 29 & $40-2$ & 293 & 0 & 0 & 293 & 11 & 0 & 304 \\
\hline 30 & $40-3$ & 310 & 0 & 0 & 310 & 4 & 0 & 314 \\
\hline 31 & $45-1$ & 291 & 0 & 0 & 291 & 5 & 0 & 296 \\
\hline 32 & $45-2$ & 355 & 0 & 1 & 354 & 4 & 0 & 358 \\
\hline 33 & $45-3$ & 355 & 1 & 0 & 354 & 6 & 0 & 360 \\
\hline 34 & $50-1$ & 374 & 2 & 0 & 372 & 5 & 0 & 377 \\
\hline 35 & $50-2$ & 405 & 3 & 0 & 402 & 9 & 0 & 411 \\
\hline 36 & $50-3$ & 577 & 0 & 0 & 577 & 9 & 0 & 586 \\
\hline 37 & $60-1$ & 530 & 1 & 0 & 529 & 5 & 0 & 534 \\
\hline 38 & $60-2$ & 496 & 1 & 0 & 495 & 10 & 0 & 505 \\
\hline 39 & $60-3$ & 547 & 0 & 0 & 547 & 0 & 0 & 547 \\
\hline 40 & $70-1$ & 686 & 1 & 0 & 685 & 4 & 0 & 689 \\
\hline 41 & $70-2$ & 777 & 3 & 4 & 770 & 56 & 1 & 827 \\
\hline 42 & $70-3$ & 778 & 0 & 1 & 777 & 12 & 0 & 789 \\
\hline 43 & $80-1$ & 1007 & 1 & 1 & 1005 & 4 & 0 & 1009 \\
\hline 44 & $80-2$ & 676 & 1 & 0 & 675 & 13 & 0 & 688 \\
\hline 45 & $80-3$ & 919 & 1 & 2 & 916 & 21 & 1 & 938 \\
\hline 46 & $90-1$ & 985 & 0 & 4 & 981 & 41 & 3 & 1025 \\
\hline 47 & $90-2$ & 969 & 6 & 1 & 962 & 26 & 0 & 988 \\
\hline 48 & $90-3$ & 1352 & 3 & 0 & 1349 & 29 & 0 & 1378 \\
\hline 49 & 100-1 & 1410 & 13 & 6 & 1391 & 38 & 1 & 1430 \\
\hline 50 & $100-2$ & 1110 & 11 & 1 & 1098 & 73 & 0 & 1171 \\
\hline 51 & $100-3$ & 1200 & 6 & 0 & 1194 & 51 & 0 & 1245 \\
\hline 52 & $125-1$ & 1624 & 4 & 5 & 1615 & 77 & 2 & 1694 \\
\hline 53 & $125-2$ & 2041 & 3 & 3 & 2035 & 42 & 0 & 2077 \\
\hline 54 & $125-3$ & 1753 & 8 & 8 & 1737 & 36 & 6 & 1779 \\
\hline 55 & 150-1 & 2726 & 12 & 13 & 2701 & 97 & 2 & 2800 \\
\hline 56 & $150-2$ & 2933 & 13 & 7 & 2913 & 168 & 5 & 3086 \\
\hline 57 & $150-3$ & 3341 & 14 & 30 & 3297 & 65 & 11 & 3373 \\
\hline 58 & 175-1 & 3261 & 4 & 22 & 3235 & 101 & 4 & 3340 \\
\hline 59 & $175-2$ & 3433 & 11 & 4 & 3418 & 76 & 0 & 3494 \\
\hline 60 & $175-3$ & 3519 & 19 & 9 & 3491 & 113 & 2 & 3606 \\
\hline 61 & 200-1 & 3786 & 8 & 9 & 3769 & 54 & 0 & 3823 \\
\hline 62 & $200-2$ & 3984 & 41 & 17 & 3926 & 331 & 1 & 4258 \\
\hline 63 & $200-3$ & 4362 & 2 & 10 & 4350 & 64 & 5 & 4419 \\
\hline
\end{tabular}


Table 15: Results H3 (Part II)

\begin{tabular}{|c|c|c|c|c|c|c|c|c|}
\hline$(10)$ & $(11)$ & $(12)$ & $(13)$ & $(14)$ & $(15)$ & $(16)$ & (17) & $(18)$ \\
\hline $\mathrm{D}$ & $C(H 3 a, H 4)$ & ND & $\mathrm{D}$ & $C(H 3 b, H 4)$ & ND & $\mathrm{D}$ & $C(H 3 a b, H 4)$ & ND \\
\hline 0 & 11 & 0 & 0 & 11 & 0 & 0 & 11 & 0 \\
\hline 0 & 8 & 0 & 0 & 8 & 0 & 0 & 8 & 0 \\
\hline 0 & 7 & 0 & 0 & 7 & 0 & 0 & 7 & 0 \\
\hline 0 & 20 & 0 & 0 & 20 & 0 & 0 & 20 & 0 \\
\hline 0 & 17 & 0 & 0 & 17 & 0 & 0 & 17 & 0 \\
\hline 0 & 16 & 0 & 0 & 16 & 0 & 0 & 16 & 0 \\
\hline 0 & 22 & 0 & 0 & 22 & 0 & 0 & 22 & 0 \\
\hline 0 & 26 & 0 & 0 & 26 & 0 & 0 & 26 & 0 \\
\hline 0 & 26 & 0 & 0 & 26 & 0 & 0 & 26 & 0 \\
\hline 0 & 30 & 0 & 0 & 30 & 0 & 0 & 30 & 0 \\
\hline 0 & 41 & 0 & 0 & 41 & 0 & 0 & 41 & 0 \\
\hline 0 & 40 & 1 & 0 & 41 & 0 & 0 & 41 & 0 \\
\hline 0 & 65 & 1 & 0 & 66 & 0 & 0 & 66 & 0 \\
\hline 0 & 39 & 0 & 0 & 39 & 0 & 0 & 39 & 0 \\
\hline 0 & 81 & 0 & 0 & 81 & 0 & 0 & 81 & 0 \\
\hline 1 & 123 & 3 & 0 & 126 & 0 & 0 & 126 & 0 \\
\hline 0 & 93 & 1 & 0 & 94 & 0 & 0 & 94 & 0 \\
\hline 1 & 121 & 7 & 0 & 128 & 0 & 0 & 128 & 0 \\
\hline 0 & 144 & 5 & 0 & 149 & 0 & 0 & 149 & 0 \\
\hline 0 & 108 & 6 & 0 & 114 & 0 & 0 & 114 & 0 \\
\hline 0 & 157 & 2 & 0 & 159 & 0 & 0 & 159 & 0 \\
\hline 0 & 219 & 4 & 0 & 223 & 0 & 0 & 223 & 0 \\
\hline 0 & 138 & 1 & 0 & 139 & 0 & 0 & 139 & 0 \\
\hline 0 & 221 & 7 & 0 & 228 & 0 & 0 & 228 & 0 \\
\hline 0 & 289 & 16 & 0 & 305 & 0 & 0 & 305 & 0 \\
\hline 0 & 274 & 0 & 0 & 274 & 0 & 0 & 274 & 0 \\
\hline 0 & 282 & 1 & 0 & 280 & 3 & 0 & 283 & 0 \\
\hline 0 & 301 & 4 & 0 & 305 & 0 & 0 & 305 & 0 \\
\hline 0 & 293 & 11 & 0 & 304 & 0 & 0 & 304 & 0 \\
\hline 0 & 310 & 4 & 0 & 314 & 0 & 0 & 314 & 0 \\
\hline 0 & 291 & 5 & 0 & 296 & 0 & 0 & 296 & 0 \\
\hline 0 & 355 & 4 & 0 & 358 & 1 & 0 & 359 & 0 \\
\hline 1 & 354 & 6 & 0 & 360 & 0 & 0 & 360 & 0 \\
\hline 2 & 372 & 5 & 0 & 377 & 0 & 0 & 377 & 0 \\
\hline 3 & 402 & 9 & 0 & 411 & 0 & 0 & 411 & 0 \\
\hline 0 & 577 & 9 & 0 & 586 & 0 & 0 & 586 & 0 \\
\hline 1 & 529 & 5 & 0 & 534 & 0 & 0 & 534 & 0 \\
\hline 1 & 495 & 10 & 0 & 505 & 0 & 0 & 505 & 0 \\
\hline 0 & 547 & 0 & 0 & 547 & 0 & 0 & 547 & 0 \\
\hline 1 & 685 & 4 & 0 & 689 & 0 & 0 & 689 & 0 \\
\hline 3 & 774 & 56 & 1 & 826 & 4 & 0 & 830 & 0 \\
\hline 0 & 778 & 12 & 0 & 789 & 1 & 0 & 790 & 0 \\
\hline 1 & 1006 & 4 & 0 & 1009 & 1 & 0 & 1010 & 0 \\
\hline 1 & 675 & 13 & 0 & 688 & 0 & 0 & 688 & 0 \\
\hline 1 & 918 & 21 & 1 & 937 & 2 & 0 & 939 & 0 \\
\hline 0 & 985 & 41 & 3 & 1022 & 4 & 0 & 1026 & 0 \\
\hline 6 & 963 & 26 & 0 & 988 & 1 & 0 & 989 & 0 \\
\hline 3 & 1349 & 29 & 0 & 1378 & 0 & 0 & 1378 & 0 \\
\hline 13 & 1397 & 38 & 1 & 1429 & 6 & 0 & 1435 & 0 \\
\hline 11 & 1099 & 73 & 0 & 1171 & 1 & 0 & 1172 & 0 \\
\hline 6 & 1194 & 51 & 0 & 1245 & 0 & 0 & 1245 & 0 \\
\hline 4 & 1620 & 77 & 2 & 1692 & 5 & 0 & 1697 & 0 \\
\hline 3 & 2038 & 42 & 0 & 2077 & 3 & 0 & 2080 & 0 \\
\hline 8 & 1745 & 36 & 6 & 1773 & 8 & 0 & 1781 & 0 \\
\hline 12 & 2714 & 97 & 2 & 2798 & 13 & 0 & 2811 & 0 \\
\hline 13 & 2920 & 168 & 5 & 3081 & 7 & 0 & 3088 & 0 \\
\hline 14 & 3327 & 65 & 11 & 3362 & 30 & 0 & 3392 & 0 \\
\hline 4 & 3257 & 101 & 4 & 3336 & 22 & 0 & 3358 & 0 \\
\hline 11 & 3422 & 76 & 0 & 3494 & 4 & 0 & 3498 & 0 \\
\hline 19 & 3500 & 113 & 2 & 3604 & 9 & 0 & 3613 & 0 \\
\hline 8 & 3778 & 54 & 0 & 3823 & 9 & 0 & 3832 & 0 \\
\hline 41 & 3943 & 331 & 1 & 4257 & 17 & 0 & 4274 & 0 \\
\hline 2 & 4360 & 64 & 5 & 4414 & 10 & 0 & 4424 & 0 \\
\hline
\end{tabular}


Table 16: Percentage of common solutions with $\mathrm{H} 4$ and CPU time (Part I)

\begin{tabular}{|c|c|c|c|c|c|c|c|c|}
\hline$(1)$ & $(2)$ & $(3)$ & $(4)$ & (5) & (6) & $(7)$ & $(8)$ & $(9)$ \\
\hline & $n$ & $|P E(H 4)|$ & $\mathrm{cpu}$ & $\% H 1 a$ & $\mathrm{cpu}$ & $\% H 1 b$ & $\mathrm{cpu}$ & $\% H 1 a b$ \\
\hline 1 & $5-1$ & 11 & & 100 & & 100 & & 100 \\
\hline 2 & $5-2$ & 8 & & 100 & & 100 & & 100 \\
\hline 3 & $5-3$ & 7 & & 100 & & 100 & & 100 \\
\hline 4 & $7-1$ & 20 & & 100 & & 100 & & 100 \\
\hline 5 & $7-2$ & 17 & & 100 & & 100 & & 100 \\
\hline 6 & $7-3$ & 16 & & 100 & & 100 & & 100 \\
\hline 7 & $9-1$ & 22 & & 100 & & 100 & & 100 \\
\hline 8 & $9-2$ & 26 & & 100 & & 100 & & 100 \\
\hline 9 & $9-3$ & 26 & & 100 & & 100 & & 100 \\
\hline 10 & $13-1$ & 30 & & 100 & & 100 & & 100 \\
\hline 11 & $13-2$ & 41 & & 100 & & 100 & & 100 \\
\hline 12 & $13-3$ & 41 & & 95.1 & & 100 & & 100 \\
\hline 13 & $15-1$ & 66 & & 98.5 & & 100 & & 100 \\
\hline 14 & $15-2$ & 39 & & 100 & & 100 & & 100 \\
\hline 15 & $15-3$ & 81 & & 100 & & 100 & & 100 \\
\hline 16 & $20-1$ & 126 & & 97.6 & & 100 & & 100 \\
\hline 17 & $20-2$ & 94 & & 98.9 & & 100 & & 100 \\
\hline 18 & $20-3$ & 128 & & 58.6 & & 100 & & 100 \\
\hline 19 & $25-1$ & 149 & & 96.6 & & 100 & & 100 \\
\hline 20 & $25-2$ & 114 & & 94.7 & & 100 & & 100 \\
\hline 21 & $25-3$ & 159 & & 98.1 & & 98.1 & & 100 \\
\hline 22 & $30-1$ & 223 & & 97.8 & & 97.3 & & 100 \\
\hline 23 & $30-2$ & 139 & & 95.0 & & 100 & & 100 \\
\hline 24 & $30-3$ & 228 & & 96.9 & & 100 & & 100 \\
\hline 25 & $35-1$ & 305 & & 94.4 & & 92.1 & & 100 \\
\hline 26 & $35-2$ & 274 & & 88.0 & & 100 & & 100 \\
\hline 27 & $35-3$ & 283 & & 54.1 & & 98.9 & & 99.3 \\
\hline 28 & $40-1$ & 305 & & 97.7 & & 100 & & 100 \\
\hline 29 & $40-2$ & 304 & & 96.1 & & 75.7 & & 100 \\
\hline 30 & $40-3$ & 314 & & 94.3 & & 100 & & 100 \\
\hline 31 & $45-1$ & 296 & & 98.0 & & 99.3 & & 100 \\
\hline 32 & $45-2$ & 359 & & 98.1 & & 78.0 & & 100 \\
\hline 33 & $45-3$ & 360 & & 96.7 & & 85.0 & & 100 \\
\hline 34 & $50-1$ & 377 & & 98.7 & & 67.1 & & 99.7 \\
\hline 35 & $50-2$ & 411 & & 96.8 & & 26.9 & & 99.8 \\
\hline 36 & $50-3$ & 586 & & 95.4 & & 42.0 & & 99.0 \\
\hline 37 & $60-1$ & 534 & & 98.3 & & 71.7 & & 99.4 \\
\hline 38 & $60-2$ & 505 & & 32.5 & & 98.4 & & 98.4 \\
\hline 39 & $60-3$ & 547 & & 96.1 & & 100 & & 100 \\
\hline 40 & $70-1$ & 689 & 1 & 89.6 & & 98.5 & & 100 \\
\hline 41 & $70-2$ & 830 & 1 & 8.4 & & 74.0 & & 82.2 \\
\hline 42 & $70-3$ & 790 & 1 & 89.1 & & 22.9 & & 98.7 \\
\hline 43 & $80-1$ & 1010 & 1 & 7.9 & & 99.4 & & 99.4 \\
\hline 44 & $80-2$ & 688 & 1 & 98.1 & & 21.7 & & 98.8 \\
\hline 45 & $80-3$ & 939 & 1 & 86.7 & & 31.2 & & 97.1 \\
\hline 46 & $90-1$ & 1026 & 5 & 23.7 & & 47.0 & & 70.5 \\
\hline 47 & $90-2$ & 989 & 5 & 9.1 & & 78.2 & & 87.1 \\
\hline 48 & $90-3$ & 1378 & 8 & 51.5 & & 37.3 & & 77.9 \\
\hline 49 & $100-1$ & 1435 & 8 & 96.3 & 1 & 82.8 & & 99.6 \\
\hline 50 & $100-2$ & 1172 & 7 & 18.8 & 1 & 24.7 & & 38.4 \\
\hline 51 & $100-3$ & 1245 & 7 & 93.7 & 1 & 47.2 & & 98.6 \\
\hline 52 & $125-1$ & 1697 & 12 & 92.6 & 1 & 38.8 & 1 & 96.4 \\
\hline 53 & $125-2$ & 2080 & 12 & 7.6 & 1 & 46.0 & 1 & 53.5 \\
\hline 54 & $125-3$ & 1781 & 9 & 92.8 & 1 & 35.7 & 1 & 97.4 \\
\hline 55 & $150-1$ & 2811 & 9 & 43.5 & 1 & 18.2 & 1 & 61.7 \\
\hline 56 & $150-2$ & 3088 & 11 & 16.4 & 1 & 10.7 & 1 & 27.0 \\
\hline 57 & $150-3$ & 3392 & 11 & 16.5 & 1 & 38.2 & 1 & 54.7 \\
\hline 58 & $175-1$ & 3358 & 16 & 14.8 & 2 & 21.7 & 1 & 36.5 \\
\hline 59 & $175-2$ & 3498 & 16 & 91.0 & 2 & 3.8 & 1 & 91.5 \\
\hline 60 & $175-3$ & 3613 & 17 & 3.8 & 2 & 11.5 & 1 & 15.3 \\
\hline 61 & 200-1 & 3832 & 17 & 12.8 & 2 & 27.6 & 1 & 40.4 \\
\hline 62 & $200-2$ & 4274 & 19 & 8.4 & 2 & 36.5 & 1 & 44.9 \\
\hline 63 & $200-3$ & 4424 & 19 & 12.4 & 2 & 18.9 & 1 & 31.2 \\
\hline
\end{tabular}


Table 17: Percentage of common solutions with $\mathrm{H} 4$ and CPU time (Part II)

\begin{tabular}{|c|c|c|c|c|c|c|c|c|c|}
\hline$(10)$ & $(11)$ & $(12)$ & $(13)$ & (14) & $(15)$ & $(16)$ & $(17)$ & (18) & (19) \\
\hline$\% H 2 a$ & $\mathrm{cpu}$ & $\% H 2 b$ & $\mathrm{cpu}$ & $\% H 2 a b$ & $\% H 3 a$ & cpu & $\% H 3 b$ & cpu & $\% H 3 a b$ \\
\hline 100 & & 100 & & 100 & 100 & & 100 & & 100 \\
\hline 100 & & 100 & & 100 & 100 & & 100 & & 100 \\
\hline 100 & & 100 & & 100 & 100 & & 100 & & 100 \\
\hline 100 & & 100 & & 100 & 100 & & 100 & & 100 \\
\hline 100 & & 100 & & 100 & 100 & & 100 & & 100 \\
\hline 100 & & 100 & & 100 & 100 & & 100 & & 100 \\
\hline 100 & & 100 & & 100 & 100 & & 100 & & 100 \\
\hline 100 & & 100 & & 100 & 100 & & 100 & & 100 \\
\hline 100 & & 100 & & 100 & 100 & & 100 & & 100 \\
\hline 100 & & 100 & & 100 & 100 & & 100 & & 100 \\
\hline 100 & & 100 & & 100 & 100 & & 100 & & 100 \\
\hline 100 & & 100 & & 100 & 97.6 & & 100 & & 100 \\
\hline 98.5 & & 100 & & 100 & 98.5 & & 100 & & 100 \\
\hline 100 & & 100 & & 100 & 100 & & 100 & & 100 \\
\hline 100 & & 100 & & 100 & 100 & & 100 & & 100 \\
\hline 97.6 & & 100 & & 100 & 100 & & 100 & & 100 \\
\hline 100 & & 100 & & 100 & 97.6 & & 100 & & 100 \\
\hline 96.1 & & 100 & & 100 & 94.5 & & 100 & & 100 \\
\hline 96.6 & & 100 & & 100 & 96.6 & & 100 & & 100 \\
\hline 100 & & 100 & & 100 & 94.7 & & 100 & & 100 \\
\hline 98.7 & & 100 & & 100 & 98.7 & & 100 & & 100 \\
\hline 97.8 & & 98.7 & & 100 & 98.2 & & 100 & & 100 \\
\hline 96.4 & & 100 & & 100 & 99.3 & & 100 & & 100 \\
\hline 99.1 & & 100 & & 100 & 96.9 & & 100 & & 100 \\
\hline 98.4 & & 99.3 & & 100 & 94.8 & & 100 & & 100 \\
\hline 94.2 & & 100 & & 100 & 100 & & 100 & & 100 \\
\hline 75.3 & & 100 & & 100 & 99.6 & & 98.9 & & 100 \\
\hline 99.3 & & 100 & & 100 & 98.7 & & 100 & & 100 \\
\hline 98.4 & & 100 & & 100 & 96.4 & & 100 & & 100 \\
\hline 94.9 & & 100 & & 100 & 98.7 & & 100 & & 100 \\
\hline 99.0 & & 99.3 & & 100 & 98.3 & & 100 & & 100 \\
\hline 98.6 & & 98.6 & & 100 & 98.9 & & 99.7 & & 100 \\
\hline 97.8 & & 100 & & 100 & 98.3 & & 100 & & 100 \\
\hline 98.7 & 2 & 97.6 & & 100 & 98.7 & & 100 & & 100 \\
\hline 97.8 & 2 & 91.0 & & 100 & 97.8 & & 100 & & 100 \\
\hline 96.1 & 1 & 86.0 & & 100 & 98.5 & & 100 & & 100 \\
\hline 98.5 & 1 & 99.4 & & 100 & 99.1 & & 100 & & 100 \\
\hline 82.6 & 1 & 100 & & 100 & 98.0 & & 100 & & 100 \\
\hline 92.1 & 1 & 100 & & 100 & 100 & & 100 & & 100 \\
\hline 89.6 & 3 & 99.0 & 1 & 100 & 99.4 & & 100 & & 100 \\
\hline 92.8 & 3 & 90.6 & 1 & 98.9 & 93.3 & & 99.5 & & 100 \\
\hline 92.7 & 5 & 96.8 & 2 & 99.9 & 98.5 & & 99.9 & & 100 \\
\hline 43.1 & 6 & 99.7 & 2 & 99.9 & 99.6 & & 99.9 & & 100 \\
\hline 98.4 & 3 & 99.6 & 1 & 100 & 98.1 & & 100 & & 100 \\
\hline 87.3 & 3 & 67.5 & 1 & 98.8 & 97.8 & & 99.8 & & 100 \\
\hline 40.7 & 13 & 79.5 & 2 & 80.8 & 96.0 & & 99.6 & & 100 \\
\hline 80.4 & 13 & 91.2 & 2 & 91.9 & 97.4 & & 99.9 & & 100 \\
\hline 55.4 & 9 & 99.6 & 4 & 99.7 & 97.9 & & 100 & & 100 \\
\hline 97.1 & 9 & 68.9 & 5 & 99.3 & 97.4 & 1 & 99.6 & & 100 \\
\hline 84.7 & 20 & 96.8 & 4 & 99.7 & 93.8 & 1 & 99.9 & & 100 \\
\hline 96.2 & 20 & 98.1 & 4 & 99.6 & 95.9 & 1 & 100 & & 100 \\
\hline 95.1 & 21 & 75.4 & 4 & 98.1 & 95.5 & 1 & 99.7 & 1 & 100 \\
\hline 22.9 & 21 & 96.9 & 4 & 96.9 & 98.0 & 1 & 99.9 & 1 & 100 \\
\hline 95.0 & 26 & 62.3 & 4 & 98.3 & 98.0 & 1 & 99.6 & 1 & 100 \\
\hline 65.7 & 26 & 60.7 & 6 & 90.9 & 96.5 & 1 & 99.5 & 1 & 100 \\
\hline 27.3 & 42 & 28.2 & 7 & 42.6 & 94.6 & 1 & 99.8 & 1 & 100 \\
\hline 20.5 & 42 & 38.5 & 7 & 54.8 & 98.1 & 1 & 99.1 & 1 & 100 \\
\hline 18.9 & 36 & 66.8 & 11 & 66.9 & 97.0 & 2 & 99.3 & 1 & 100 \\
\hline 91.0 & 36 & 41.1 & 11 & 93.6 & 97.8 & 2 & 99.9 & 1 & 100 \\
\hline 7.9 & 49 & 69.3 & 13 & 70.9 & 96.9 & 2 & 99.8 & 1 & 100 \\
\hline 13.2 & 49 & 32.4 & 13 & 42.6 & 98.6 & 2 & 99.8 & 1 & 100 \\
\hline 9.7 & 35 & 52.0 & 16 & 52.1 & 92.3 & 2 & 99.6 & 1 & 100 \\
\hline 25.3 & 35 & 97.4 & 16 & 97.6 & 98.6 & 2 & 99.8 & 1 & 100 \\
\hline
\end{tabular}


Table 18: Synthetic view of the results: average $\%$

\begin{tabular}{|c|c|c|c|c|c|c|c|c|c|}
\hline$n$ & $H 1 a$ & $H 1 b$ & $H 1 a b$ & $H 2 a$ & $H 2 b$ & $H 2 a b$ & $H 3 a$ & $H 3 b$ & $H 3 a b$ \\
\hline $5-7-9$ & 100 & 100 & 100 & 100 & 100 & 100 & 100 & 100 & 100 \\
\hline $13-15-20$ & 94.30 & 100 & 100 & 99.13 & 100 & 100 & 98.56 & 100 & 100 \\
\hline $25-30-35$ & 90.60 & 98.48 & 99.92 & 95.30 & 99.77 & 100 & 97.64 & 99.87 & 100 \\
\hline $40-45-50$ & 96.86 & 78.22 & 99.83 & 97.62 & 96.94 & 100 & 98.25 & 99.96 & 100 \\
\hline $60-87-800$ & 66.30 & 68.64 & 97.11 & 83.64 & 94.73 & 99.72 & 98.20 & 99.90 & 100 \\
\hline $90-100-125$ & 53.45 & 48.63 & 79.93 & 74.16 & 85.41 & 96.03 & 96.65 & 99.80 & 100 \\
\hline $150-175-200$ & 24.40 & 20.78 & 44.80 & 31.05 & 54.04 & 68.00 & 96.71 & 99.64 & 100 \\
\hline All instances & 75.13 & 73.53 & 88.79 & 83.37 & 90.12 & 94.82 & 98.00 & 99.88 & 100 \\
\hline
\end{tabular}

